\title{
Quantitative Systematic Analysis of Vocal Tract Data
}

\author{
Samuel Silva ${ }^{\mathrm{a}, \mathrm{b}, *}$, António Teixeira ${ }^{\mathrm{a}, \mathrm{b}}$ \\ ${ }^{a}$ Dep. Electronics, Telecommunications and Informatics (DETI), \\ University of Aveiro, \\ Aveiro, Portugal \\ ${ }^{b}$ Inst. Electronics Eng. and Telematics of Aveiro (IEETA), \\ University of Aveiro, \\ Aveiro, Portugal
}

\begin{abstract}
Articulatory data can nowadays be obtained using a wide range of techniques, with a notable emphasis on imaging modalities such as ultrasound and realtime magnetic resonance, resulting in large amounts of image data.

One of the major challenges posed by these large datasets concerns how they can be efficiently analysed to extract relevant information to support speech production studies. Traditional approaches, including the superposition of vocal tract profiles, provide only a qualitative characterisation of notable properties and differences. While providing valuable information, these methods are rather inefficient and inherently subjective. Therefore, analysis must evolve towards a more automated, replicable and quantitative approach.

To address these issues we propose the use of objective measures to compare the configurations assumed by the vocal tract during the production of different sounds. The proposed framework provides quantitative normalized data regarding differences covering meaningful regions under the influence of various articulators. An important part of the framework is the visual representation of the data, proposed to support analysis, and depicting the differences found and corresponding direction of change.

The normalized nature of the computed data allows comparison among
\end{abstract}

\footnotetext{
${ }^{*}$ Corresponding author: Samuel Silva, IEETA, Campus Univ. de Santiago, 3810-193 Aveiro, Portugal

Email addresses: sss@ua.pt (Samuel Silva), ajst@ua.pt (António Teixeira)
} 
different sounds and speakers in a common representation.

Representative application examples, concerning the articulatory characterisation of European Portuguese vowels, are presented to illustrate the capabilities of the proposed framework, both for static configurations and the assessment of dynamic aspects during speech production.

Keywords: vocal tract analysis, quantitative comparison, RT-MRI

\section{Introduction}

Speech production studies are currently served by a wide range of technologies that allow research on the dynamic aspects of speech. Methods such as ultrasound (US) and real-time magnetic resonance imaging (RTMRI) (Scott et al., 2014) provide data regarding the position and coordination of the different articulators over time (Hagedorn et al., 2011). Furthermore, they offer the possibility to improve on the studies based on information regarding a static sustained production by reducing the hyperarticulation effect (Engwall, 2003).

After image acquisition, the different regions of interest must be segmented (e.g., Bresch and Narayanan, 2009; Silva and Teixeira, 2015), or points of interest identified, often resulting in contours delimiting the vocal tract or specific structures such as the tongue or velum.

Analysis of different vocal tract contours is typically performed visually by characterizing the position of the different articulators or by describing articulator differences between different sounds (e.g., Delvaux et al., 2002; Shadle et al., 2008). This is often done by superimposing contours and performing qualitative analysis of the main differences (Martins et al., 2008; Cleland et al., 2011; Badin et al., 2014). Adding to the subjective nature of such analysis, when the database is large, e.g., as happens when RT-MRI is used (Niebergall et al., 2013), it becomes an almost infeasible task to explore all available data.

\subsection{Challenges}

Beyond the sheer amount of data made available by current technologies, the field of speech production faces several challenges that should be addressed to allow further advances, harnessing the full potential of the data available. A framework should be proposed that tackles the large amounts of data addressing, among others, the following aspects: 
Objectivity - The subjective nature of the methods used to describe articulatory differences, for example, results in variability among researchers that precludes true comparison among works in the literature describing the same phenomena;

Intra-speaker assessment - The analysis of articulatory features for different sounds produced by one speaker lacks methods to profit from multiple repetitions and common grounds for comparison among sounds;

Inter-speaker assessment - The comparison among speakers lacks common grounds for comparison, e.g., a common normalized measure of difference, without losing sight of the contributions provided by each articulator.

Variability - Not only average behaviour is relevant for the researchers, there is also a strong need to have information on variability (across repetitions, across speakers, etc.).

Inter-language comparison - data from multiple speakers of one language could be jointly used to provide overall quantitative characterisation of its main features. This would allow new ways of comparing sounds in multiple languages, advancing on the current status of interlanguage comparisons mostly based on qualitative assessment of data from different speakers;

Multimodality - data provided by different modalities and concerning similar phenomena or providing complementary data might benefit from joint analysis. For example, several technologies that support speech production studies (e.g., EMA, Kim et al. 2014 and ultrasound, Laprie et al. 2014) are used in combination with MRI (Scott et al., 2014). Regardless of how the different data is analysed, if their individual contributions to the understanding of specific phenomena could be gathered in joint representations it might motivate a generalization of multimodal studies and an easier interpretation of the data.

One important route to attain a systematic analysis addressing these issues is to move towards quantitative methods that allow it to be performed automatically, in an expedite and replicable way, resulting in data providing a summary of the most important features which researchers can analyse. In the work presented here we consider these challenges in the scope of real-time MRI data. 


\subsection{Related Work}

Considering that we are mostly addressing vocal tract data analysis from vocal tract profiles extracted from image data (e.g., RT-MRI), in this overview of related work we focus on methods applied to image data of the vocal tract, or to full (or partial) vocal tract profiles extracted from them. The rationale is to overview notable recent literature that describes some level of quantification regarding the analysis and comparison of the data extracted from these images. Therefore, works supported on subjective analysis based, for example, on visual assessment of articulatory configuration differences, are not covered.

Regarding pixel-based methods, i.e., without an explicit extraction of vocal tract profiles, notable works include the detection of constriction regions along the tract (Lammert et al., 2010; Hagedorn et al., 2011) and estimation of articulator trajectories (Proctor et al., 2011; Shosted et al., 2012) contributing to improved analysis of articulatory configurations, articulatory gestures and their coordination.

Other authors have performed quantitative comparison of specific regions/articulators, the most common being the tongue. Davidson (2006) compare tongue contours using smoothing splines ANOVA (Wang, 2011). The use of Bayesian confidence intervals allows a distinction of which parts of the tongue contour are statistically different, but fails to quantify the extent of the difference between the curves (Zharkova and Hewlett, 2009). In Teixeira et al. (2011) tongue contours are compared using the Pratt index (Pratt, 2007). In the work by Zharkova et al. (2014) tongue contours are compared by computing the Euclidean distances between each point in one contour to the nearest point in the other and vice-versa and averaging the sum of all computed distances.

When considering the whole vocal tract, Proctor et al. (2010) and Shosted et al. (2012), for example, have extracted distance functions, which might not be a clear way to detect which articulator has the most influence.

On a different level, Miller et al. (2014) gather vocal tract configuration data (also including the cervical spine and airway), from multiple speakers, by building an active shape model (ASM). Through the identification of independent modes of variation, and selecting those that account for the most part of the explained variance, the authors study coordinated changes affecting the cervical spine, airway and vocal structures. One interesting idea to note concerns gathering the data for all speakers to reach a quantitative measure of mean behaviour and variability, which allows the characterisation 
of the articulatory space defined by the vocal tract configurations used for training. Comparison among vocal tract profiles might eventually be performed based on the weights of each of the modes of variation, but with the inherent difficulties in isolating the contribution of individual articulators. Furthermore, the resulting ASM model depends on the images used to train it and, therefore, a comparison cannot be performed with weights obtained for models resulting from different training sets.

Ramanarayanan et al. (2010, 2013) have proposed a set of vocal tract area descriptors, analogous to the distance functions, by dividing the vocal tract in clearly defined regions considering lip and velum aperture and the constriction locations at tongue tip, dorsum and root. The authors compute the area contained in the vocal tract regions delimited by the above locations and use these area descriptors to compare among vocal tract postures (Benítez et al., 2014). While it does not exactly provide information on specific articulators it allows a slightly better grasp of overall localisation than with distance functions.

On the subject of inter-speaker or inter-language comparison and regarding specific articulators, Zharkova et al. (2011) normalized measures performed over tongue contours (to compare between pre-adolescents and adults) by computing the tongue's length for specific tongue postures.

To allow direct comparison among different speakers, accounting for the morphological differences, vocal tract normalization has also been proposed by several authors. A common approach is the normalization of vocal tract length and the presentation of distance functions (e.g., Martins et al., 2008; Proctor et al., 2010). As alluded before, the use of distance functions, even though providing a good overall idea of the differences among speakers does not explicitly address particular articulators. A normalization of area descriptors of the vocal tract has also been performed, e.g., by Benítez et al. (2014), with advantages over distance functions, due to an association between each descriptor and a region of the vocal tract.

Badin et al. (2014) use articulatory modelling to compare between classes of sounds produced by one speaker for two languages. By using data from vowels, analogous consonants and the full corpus, obtained using MRI, articulatory models are created. While this provides a quantitative method to gather the different data and characterise the articulatory space for each class of sounds, involving a selection of the variation modes accounting for a large percentage of the explained variance, the comparison is still performed by varying model parameters, in specific ranges, and visually comparing the 
resulting model configuration. The authors propose to evaluate how the articulatory spaces from both languages overlap by using the models built for one language to reconstruct the articulations collected for the other. Nevertheless, it is still not clear how this procedure can be used to draw any conclusion since the models are built for more than one sound and are, therefore, naturally capable of encompassing some variability.

Overall, while several methods have been proposed to support quantitative analysis and comparison of vocal tract data, there are no clear approaches to systematically handling the large amounts of data available from imaging technologies, such as RT-MRI, to take advantage of multiple realisations of each sound and explicitly supporting the comparison within and among speakers and languages.

\subsection{Contributions and Overview}

The pipeline in figure 1 shows the main steps associated with image-based speech production studies, taking into consideration what might be at stake to address the challenges identified above, comprising: data acquisition (e.g., using RT-MRI); feature extraction such as vocal tract segmentation (e.g., Bresch and Narayanan, 2009; Silva and Teixeira, 2015); comparison of features between vocal tract profiles; and an additional step regarding computational analysis. This last step can build on the data computed for vocal tract configuration differences, apply more advanced analysis methods, such as cluster analysis, and will depend on the nature of the data made available by the preceding step.

Considering the literature surveyed above, and to the best of our knowledge, no method has been proposed to support a more complete vocal tract profile comparison, providing meaningful data regarding the regions under the influence of the different articulators and addressing the different challenges posed to current speech production studies.

To address these aspects, dealing with the analysis of such data, as depicted at the centre of the pipeline presented in figure 1, and following on work presented in (Silva et al., 2013b; Silva and Teixeira, 2014c,b), we propose a framework which, among others, provides the following notable features:

- Quantitative comparison of vocal tract profiles considering the regions under the influence of different articulators;

- Normalized differences allowing intra- and inter-speaker comparisons; 


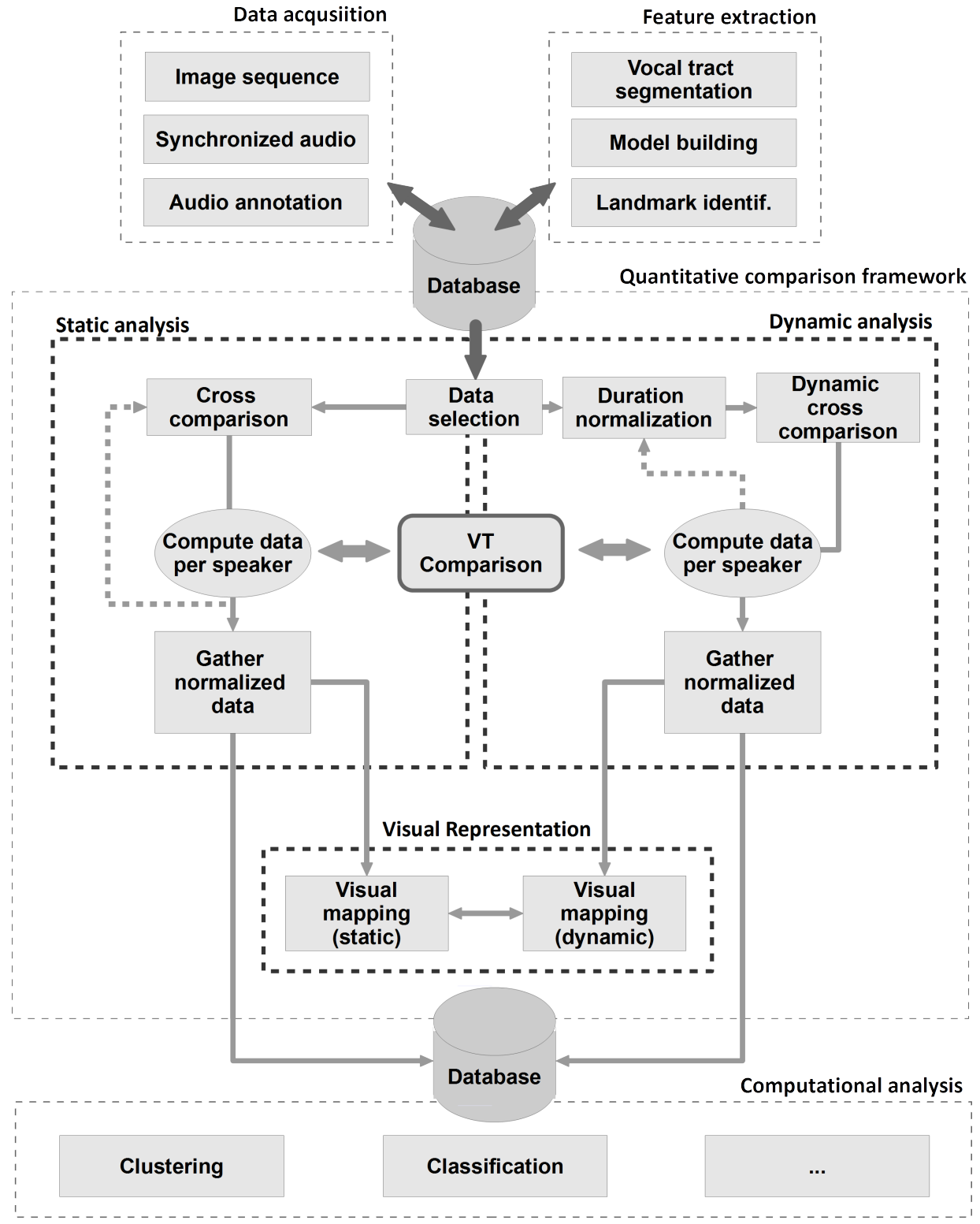

Figure 1: Pipeline depicting the main steps involved in performing speech production analysis based on image data. At the centre, in more detail, the analysis step, the focus of this work. 
- Analysis of dynamic aspects of speech production by gathering data from multiple speakers and considering multiple realisations for each sound or utterance;

- Abstract representation to support analysis and comparison of the computed difference data.

To illustrate, on a real scenario, the potential and different applications made available by the proposed methods, examples are presented dealing with data from an RT-MRI dataset collected to study the nasal vowels in European Portuguese (Teixeira et al., 2012).

The remainder of this paper is organised as follows: section 2 provides a description of the proposed methods for vocal tract comparison; section 3 presents the abstract representation built to depict the comparison data; section 4 extends the vocal tract comparison method to the analysis of dynamic aspects of speech production; section 5, describes the application of the proposed methods to data gathered in an RT-MRI study involving European Portuguese (EP) oral and nasal vowels, providing a brief illustration of their application in a real scenario; section 6 briefly discusses the work carried out; finally, section 7 presents conclusions and ideas for further work.

\section{Vocal Tract Configuration Comparison}

Vocal tract comparison is traditionally performed to assess articulatory differences among sounds and speakers. While the analysis for each speaker is usually performed by superimposing vocal tract profiles, the comparison among speakers is performed by comparing these superpositions side-by-side: since the anatomy for each speaker is different it is not possible to extract meaningful data by superposing vocal tract contours from different speakers. Figure 2 presents some examples of different pairs of superimposed contours for different speakers and sounds. Notice, for example, the subjectivity inherent to the judgement of what is a meaningful (or comparably large) difference and the difficulty to apply the same criteria when the analysis is performed by different researchers (e.g., in different studies).

The framework to support quantitative analysis of differences among vocal tract profiles (centre blocks in figure 1) is grounded on three main blocks:

- Static analysis, dealing with the comparison between vocal tract profiles deemed representative of the configurations assumed to produce 

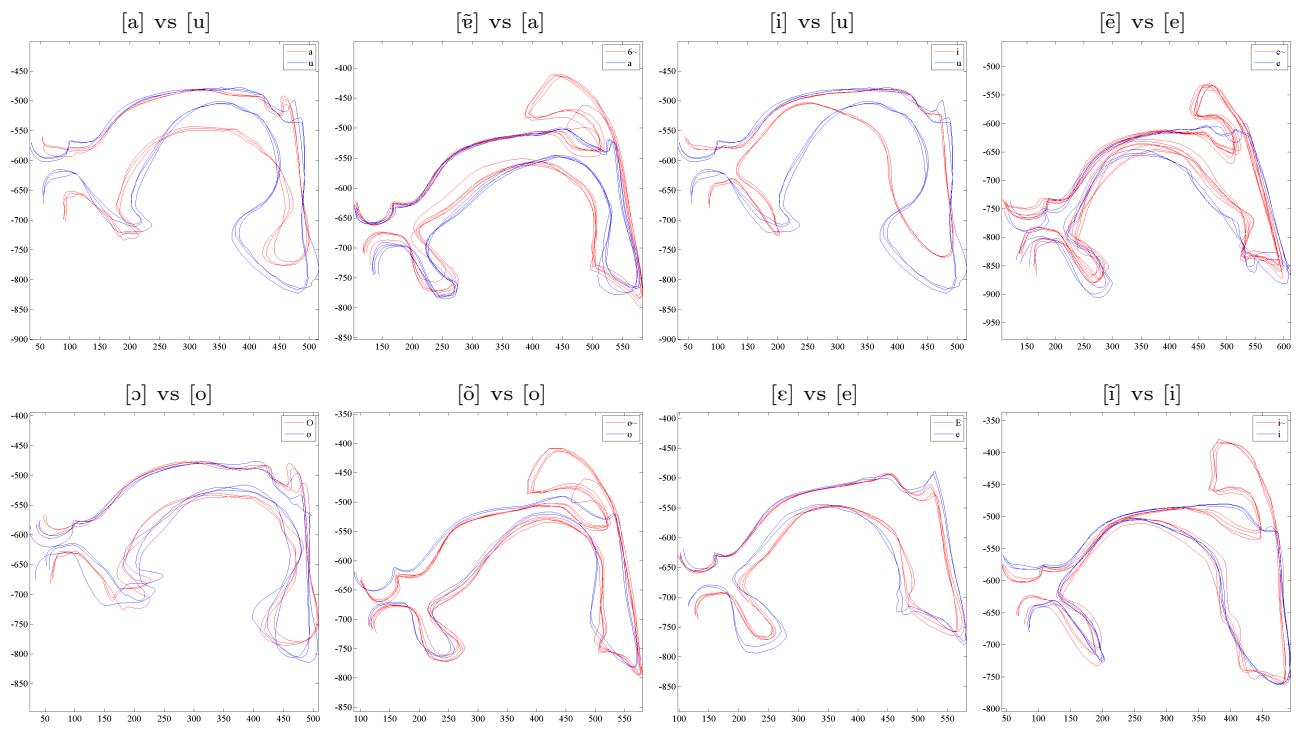

Figure 2: Examples of contour superpositions used to assess the differences between the configurations assumed by the vocal tract to produce various sounds.

different sounds (e.g., representative image frames of the vocal tract

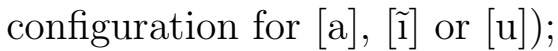

- Dynamic analysis, dealing with the comparison between the configurations assumed by the vocal tract along utterances and conveying the differences along time;

- Visual representation, concerning the depiction of the quantitative data computed in the analysis blocks using abstract representations to support interpretation of the data and dissemination.

Vocal tract comparison occupies a central role in the proposed analysis framework (figure 1) as it provides the grounds for all the provided analysis features. In what follows we set the requirements for a vocal tract comparison method, describe the adopted terminology, discuss a first set of considered regions of interest and define the normalized measures proposed to compare them between vocal tract profiles.

\subsection{Requirements and Definitions}

To tackle the challenges faced by speech production studies, discussed in the introduction, the vocal tract comparison method must go beyond current 
practice towards an objective measure of difference that can be systematically applied to the increasingly large real-time datasets. As a first set of requirements, the differences between vocal tract profiles should be:

- Obtained using objective, comparable measures;

- Computed for the different anatomical regions of interest and/or vocal tract features to provide a meaningful regional measure of difference;

- Normalized to allow comparison among different speakers and languages;

- Informative on how the difference occurred (e.g., in what direction did the tongue back move);

- Represented visually to help users understand the resulting data and support results dissemination.

Before advancing to a detailed definition of the comparison method, and in order to set a terminology to be used throughout the remainder of this article, let us consider each vocal tract contour, $C_{i}$, defined as a set of points in 2D:

$$
C_{i}=\left\{\left(x_{i 1}, y_{i 1}\right),\left(x_{i 2}, y_{i 2}\right) \ldots,\left(x_{i M}, y_{i M}\right)\right\}
$$

where $M$ is the number of points $(x, y)$ in the contour and can vary among contours.

For each contour, a fixed number of segments of interest, $N_{S}$, can be considered

$$
C_{i}=\left\{S_{i 1}, S_{i 2}, \ldots, S_{i N_{S}}\right\}, N_{S} \leq M,
$$

where each segment is defined as a subset of contour points of size $1<l_{s}<M$, as given by

$$
S_{s}=\left[\left(x_{w_{s}}, y_{w_{s}}\right)\left(x_{\left(w_{s}+l_{s}\right)}, y_{\left(w_{s}+l_{s}\right)}\right], w_{s}=\sum_{i=1}^{s-1} l_{i}\right.
$$

Each segment of interest identifies a relevant part of the vocal tract such as the tongue, the hard palate or the degenerate case of a single point (landmark). After identification of the segments of interest, a set of $N_{F}$ features to consider for comparison between vocal tract profiles can be chosen. A 
feature can be associated to a particular segment or can rely on multiple segments for its computation. For example, lip aperture needs the segments corresponding to both lips.

The feature context $\left(F^{\prime}\right)$, i.e., the relevant data that is needed to compute and compare each feature between vocal tract profiles, can then be defined based on the segments of interest. As remarked, each feature might depend on a variable number of segments:

$$
F_{f}^{\prime}=\left\{S_{i a}, S_{i b}, \ldots\right\}, a, b \in\left[1 . . N_{S}\right]
$$

The difference between two contours, $C_{i}$ and $C_{j}$, can be expressed as

$$
D\left(C_{i}, C_{j}\right)=\left\{\mathbf{F}_{\mathbf{1}}\left(F_{i 1}^{\prime}, F_{j 1}^{\prime}\right), \mathbf{F}_{\mathbf{2}}\left(F_{i 2}^{\prime}, F_{j 2}^{\prime}\right), \ldots, \mathbf{F}_{\mathbf{N}_{\mathbf{F}}}\left(F_{i N_{F}}^{\prime}, F_{j N_{F}}^{\prime}\right)\right\}
$$

with $0 \leq \mathbf{F}_{\mathbf{f}}(A, B) \leq 1$ denoting the normalized difference computed for feature $f$ and defined individually according to the feature characteristics.

\subsection{Segments and Features of Interest}

At this stage, our purpose was to provide a first set of features that could cover the whole vocal tract. To select the segments and features considered for comparing vocal tract profiles we considered the speech production studies literature and the relevance given to the different articulators and landmarks.

Considering the vocal tract, the literature (e.g., Bresch and Narayanan, 2009) identifies multiple anatomical regions with relevance for speech production, namely: upper lip, lower lip, tongue, tongue tip, velum, hard palate, pharyngeal wall, epiglottis and glottis.

Regarding relevant features extracted from the vocal tract, notable examples in the literature (Saltzman and Munhall, 1989; Gick et al., 2004; Bresch et al., 2010; Niebergall et al., 2013) include: lip protrusion; lip aperture; tongue tip constriction degree, defined by the distance from the tongue tip to the alveolar ridge; tongue dorsum constriction location and tongue dorsum constriction degree, based on the point of minimum distance between the tongue dorsum and the hard palate; tongue root constriction degree, obtained from the distance between the tongue root and the pharyngeal wall; and velar aperture.

The literature regarding the characterisation of articulatory differences (typically from contours) also provides useful information regarding which 


\begin{tabular}{lll}
\hline Feature & Notation & Feature Context $\left(F^{\prime}\right)$ \\
\hline Tongue back & $\mathbf{F}_{T B}$ & tongue back \\
Tongue dorsum & $\mathbf{F}_{T D}$ & tongue dorsum \\
Velum position & $\mathbf{F}_{V E L}$ & velum \\
Tongue tip position & $\mathbf{F}_{T T}$ & tongue tip, alveolar ridge \\
Lip protrusion & $\mathbf{F}_{L P}$ & upper lip, lower lip, alveolar ridge \\
Lip aperture & $\mathbf{F}_{L A}$ & upper lip, lower lip, alveolar ridge \\
Pharynx & $\mathbf{F}_{P h}$ & pharynx \\
\hline
\end{tabular}

Table 1: Features considered to compare vocal tract profiles, abbreviated name and vocal tract segments used to compute them.

aspects are the most important in vocal tract configuration characterisation: tongue height and backness, lip aperture and protrusion and velum height.

Considering the different features described in the literature we aimed to choose a set that would cover the whole vocal tract and provide data regarding the aspects described and used the most, by researchers, to differentiate among vocal tract configurations. In this first instantiation of the framework, the considered features for comparison are: tongue back differences, tongue dorsum differences, velum differences, tongue tip position variation, lip protrusion variation, lip aperture variation and pharynx configuration differences.

Table 1 presents a summary of the considered features, the adopted notation and segments considered as part of their feature context. The alveolar ridge is included in the context of various features because it is used for normalization purposes, as explained ahead. The difference between two vocal tract profiles can, therefore, be expressed as a features vector:

$$
D\left(C_{i}, C_{j}\right)=\left\{\mathbf{F}_{T B}, \mathbf{F}_{V E L}, \mathbf{F}_{T D}, \mathbf{F}_{T T}, \mathbf{F}_{L P}, \mathbf{F}_{L A}, \mathbf{F}_{P h}\right\}
$$

It is important to make it clear that in the context of our work we are not currently dealing with intrinsic features, i.e., features that are defined only considering one vocal tract profile. All features should be understood in the context of a comparison between two vocal tract profiles. For example, tongue backness, $\mathbf{F}_{T B}$, is not an absolute measure of the tongue back position towards the pharyngeal wall, but the comparison between the position of the tongue backs in two vocal tract configurations.

Please also note that the consideration of this set of features should not 
be understood as a restriction of the framework to it. By definition, this set is extendible to other features, as long as they abide to the requirements described in 2.1 .

The details regarding how each of the selected features is computed are provided in the following section.

\subsection{Comparison Methods}

When performing the comparison for the multiple features defined above, three different types of comparisons need to be performed: 1) between segments of the vocal tract (e.g., tongue dorsum); 2) between landmarks (e.g., tongue tip); or 3) between distances (e.g., lip aperture). Therefore, for each particular situation, a different comparison approach is needed. It should be noted that comparison measures are always computed between vocal tracts of the same speaker, considering they are already aligned, e.g., as a result of the acquisition procedure, or post acquisition alignment. Figure 3 illustrates the different vocal tract regions that need to be compared and relevant aspects of their computation.

The pharynx, $\left(\mathbf{F}_{P h}\right)$, tongue back, $\left(\mathbf{F}_{T B}\right)$, tongue dorsum $\left(\mathbf{F}_{T D}\right)$ and velum $\left(\mathbf{F}_{V E L}\right)$ are part of the features that involve the comparison between contour segments. These are compared by computing the Pratt index for each pair of corresponding contours and given by Pratt (2007):

$$
P=\frac{1}{N} \sum_{i=1}^{N} \frac{1}{1+\alpha d_{i}^{2}}
$$

where $N$ is the number of corresponding points between the compared contour segments (e.g., tongue back), $d_{i}$ is the Euclidean distance between two corresponding points, and $\alpha$ is a constant set to $1 / 9$, based on Pratt's work and similar works in the literature (Pratt, 2007). At this stage, the same constant value has been used for all regions, but it might be tuned for each region if different sensibilities to differences are desired. To obtain corresponding points between segments, the segment with the smallest number of points is selected and, for each point, the closest point in the other segment is considered the corresponding point. The Pratt's figure of merit provides values in the range $] 0,1]$ where 1 is attained when there are no differences between the countour segments.

The tongue tip position variation, $\mathbf{F}_{T T}$, is the only feature that deals with the comparison of two landmarks. Comparison is performed by computing 


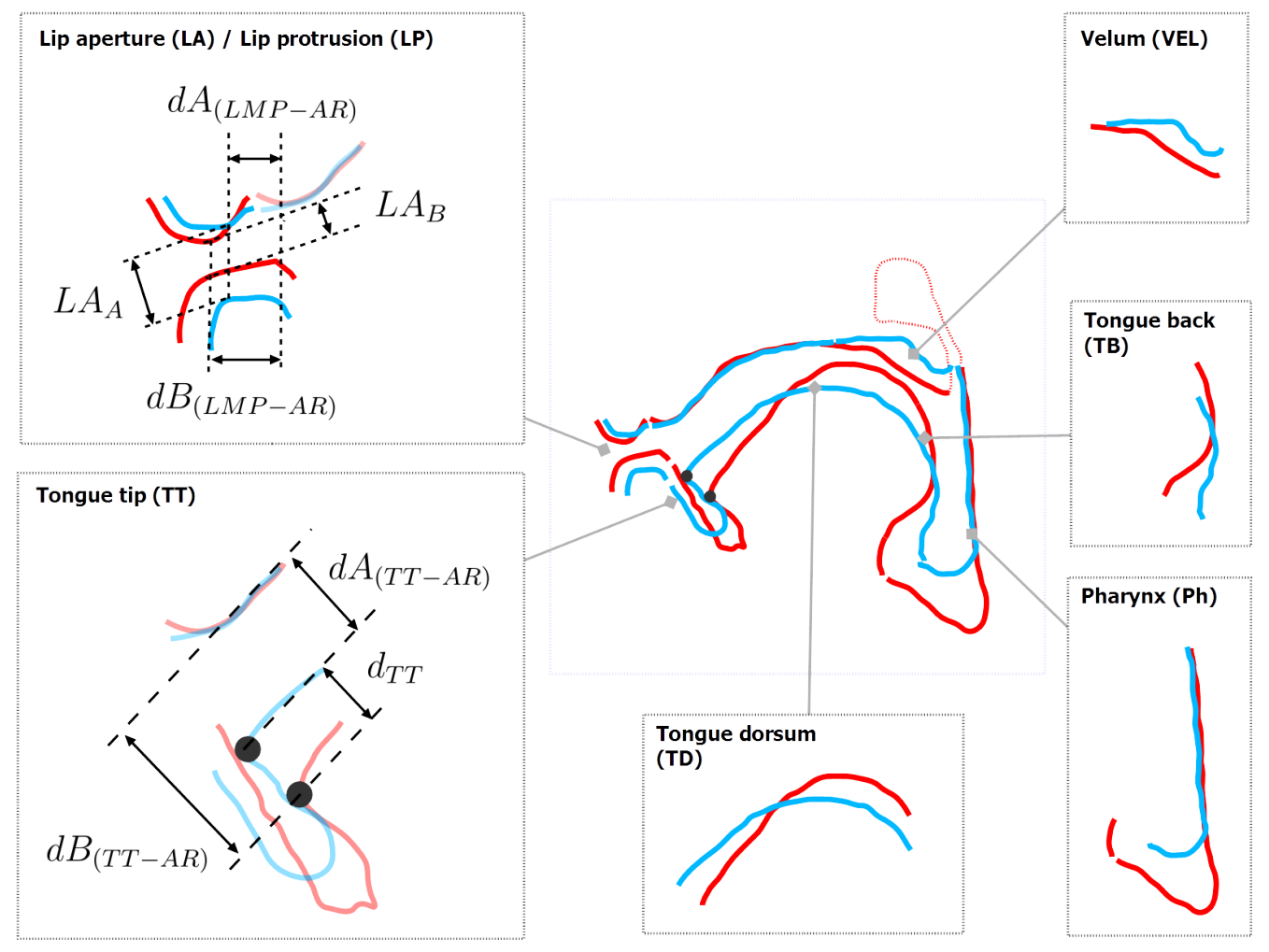

Figure 3: Illustration of the different aspects involved in computing the difference between two vocal tract profiles. Please refer to the text for additional details on how the different features are computed. 
the distance between the tongue tips $\left(d_{T T}\right)$, in both contours, and normalized by the longest distance from each tongue tip to the alveolar ridge (AR) between the two contours. Figure 3 depicts the values considered and used as follows:

$$
\mathbf{F}_{T T}=1.0-\frac{d_{T T}}{\max \left(d A_{(T T-A R)}, d B_{(T T-A R)}\right)} .
$$

The remaining two features belong to the third type and deal with the comparison between two distances that are first computed from each of the vocal tracts. Lip protrusion, $\mathbf{F}_{L P}$, is obtained (refer to figure 3 ) by computing the horizontal displacement of the mid-point between the upper and lower lips, MPL. The mid-point is computed considering the line that connects the lowest point of the upper lip with the highest point of the lower lip. To perform normalization, the horizontal distance between the mid-points between the lips and the alveolar ridge $(\mathrm{AR})$ is obtained $\left(d A_{(M P L-A R)}\right.$ and $\left.d B_{(M P L-A R)}\right)$ and used as follows (fig. 3):

$$
\mathbf{F}_{L P}=1.0-\frac{\left|d B_{(M P L-A R)}-d A_{(M P L-A R)}\right|}{\max \left(d A_{(M P L-A R)}, d B_{(M P L-A R)}\right)} .
$$

Lip aperture, $\mathbf{F}_{L A}$, is computed based on the lip aperture values for both vocal tract profiles $\left(L A_{A}, L A_{B}\right)$ and is normalized by considering the longest of the two:

$$
\mathbf{F}_{L A}=1.0-\frac{\left|L A_{B}-L A_{A}\right|}{\max \left(L A_{A}, L A_{B}\right)} .
$$

The features described above still do not provide any information regarding the direction in which the difference occurred (e.g., did the tongue tip go up or down). For each of the features the direction can be computed as the vector characterising the movement of the centre of gravity of the feature context (excluding the alveolar ridge, where present) or the difference between notable points (e.g., the midpoint between the lips in $\mathbf{F}_{L P}$. The obtained directional information is processed to provide only the component of interest. For example, for the tongue back there is no particular interest in the vertical component of displacement and the opposite happens for the tongue dorsum for which the horizontal displacement, if exists, has no major relevance.

\subsection{Taking Advantage of Repeated Utterances}

It is common that the corpora acquired during speech production studies include repetitions of each sound considered. Therefore, analysis should con- 
sider these repetitions when comparing between sounds by cross comparing all occurrences of each sound. Figure 4 illustrates the comparison between two sounds. The relevant repetitions of each sound are considered and cross compared using the methods previously described. From each of these comparisons results a set of difference values covering each of the considered regions. The average values are obtained along with the standard deviation and used as representative of the comparison between the two sounds.

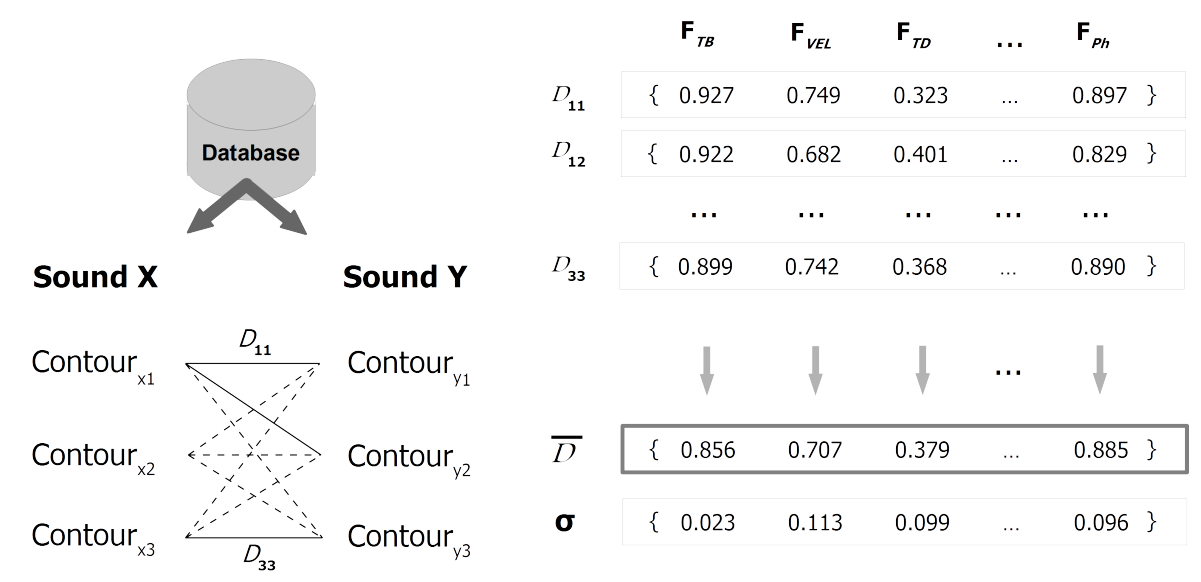

Figure 4: Comparison between two sounds taking into account existing repetitions. The contours are cross-compared between the sounds and the average difference, $\bar{D}$, and standard deviation, $\sigma$, computed.

\section{Visual Representations}

The quantitative comparison method presented above, resulting in a set of numerical values, demands a visualization method that can help users to interpret/compare and disseminate them in an easy way as opposed, for example, to value tables. The adoption of a visual representation for the data, to improve how it can be read, should not be understood as the 'end of the line' for the gathered numerical data. It will serve to provide users with an insight over the numbers, but additional methods (e.g., cluster analysis) can be applied to further explore them as depicted in the pipeline of figure 1.

One possible representation would be to present the values in a line plot, with the parameters shown in a fixed order, in the X-axis. Nevertheless, we considered that a different representation might bring easier interpretation if, 
somehow, one could build some analogy with the vocal tract and the position of the different articulators.

Figure 5 illustrates the construction of the proposed representation. The computed comparison values are represented over the unitary circle in a radar-like representation and its design is grounded in the ideas described in what follows.

\subsection{Parameter Location}

First of all, we consider the traditionally adopted orientation for speech production images with the lips presented to the left (e.g., Höwing et al., 1999; Ramanarayanan et al., 2013). The location of each computed parameter in the representation is chosen as if the vocal tract was inscribed in the unitary circle and selecting, at each time, the parameter concerning the closest region or articulator. Furthermore, the orientation associated with each value is, where applicable/possible, related with its movement direction (Niebergall et al., 2013). Accounting for these criteria, tongue back differences, for example, should be on the opposite side regarding lip protrusion. Starting at zero degrees, for the tongue back $\left(\mathbf{F}_{T B}\right)$, it follows the velum $\left(\mathbf{F}_{V E L}\right)$, tongue dorsum $\left(\mathbf{F}_{T D}\right)$, tongue tip $\left(\mathbf{F}_{T T}\right)$, lip protrusion $\left(\mathbf{F}_{L P}\right)$, lip aperture $\left(\mathbf{F}_{L A}\right)$ and pharynx $\left(\mathbf{F}_{P h}\right)$.

In the case of lip aperture, the direction used in the representation is not directly related with the direction of the expected difference. Notice, however, that considering the two parameters associated with the lips, $\mathbf{F}_{L P}$ and $\mathbf{F}_{L A}$, the directions adopted for the pair resemble an open mouth, with $\mathbf{F}_{L A}$ as the jaw.

Each feature name is presented on the exterior of the circle close to its corresponding orientation.

\subsection{Value Representation}

The values obtained for each of the considered features are represented over the corresponding direction in the unitary circle using a dot. Since it is the unitary circle, with the origin at its centre, the closest the dot is to the centre, the lowest the value it represents. As the represented value gets closer to 1.0, the dot gets closer to the circumference of the unitary circle.

\subsection{Difference Level}

To help on the interpretation of the presented values, three circular coronas are proposed. The first, between 0.75 and 1.0, in green, is suggested to 


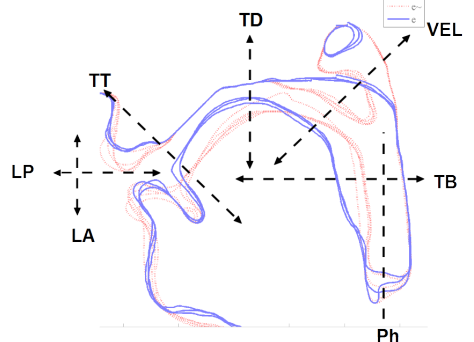

(a)

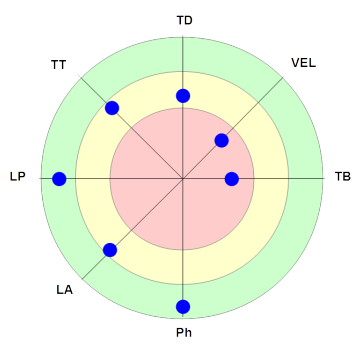

(d)

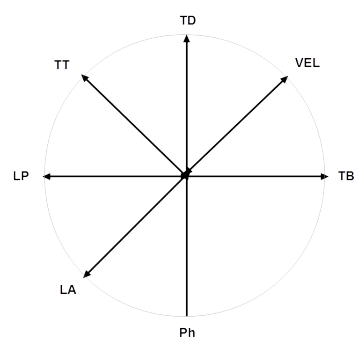

(b)

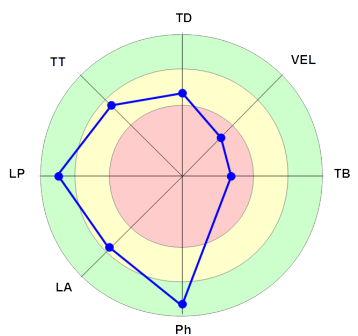

(e)

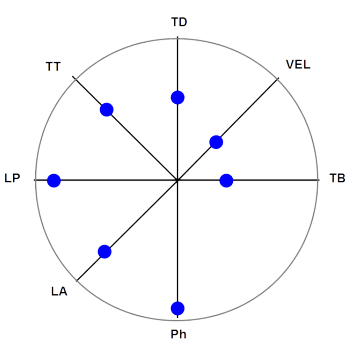

(c)

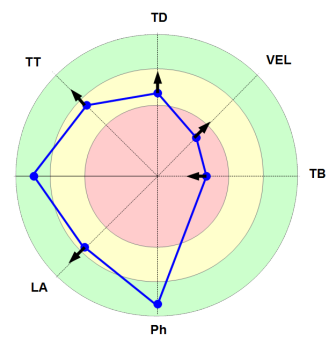

(f)

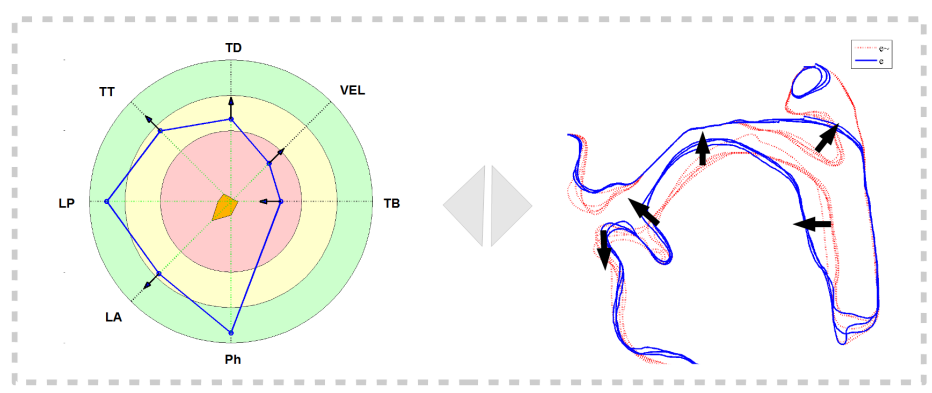

(g)

Figure 5: Different steps involved in building the abstract representation for comparison data: a) different orientations are considered for the chosen features; b) each feature is assigned an orientation over the unitary circle; c) the value computed for each feature is represented by a dot along the corresponding orientation; d) circular coronas are added to easy interpretation; e) the different dots are connected by lines, forming a polygon; $f$ ) for dots in the yellow and red regions, directional information is added; and g) abstract representation and corresponding vocal tract contours side-by-side. 
mean no relevant difference. The interval lower bound is chosen based on the value typically interpreted as meaning a good match between contours compared using the Pratt index. The second circular corona, between 0.50 and 0.75 is proposed to be interpreted as a mild difference and the last, from 0.0 to 0.50 , as a strong difference.

These are proposed interpretations and different criteria can be adopted, but their intent is to help users to have common interpretation criteria and to move away from direct numerical comparison which would lead, yet again, to a rather subjective assessment. Furthermore, the intent is also not to have too much difference levels.

\subsection{Overall Interpretation}

As a first approach, trying to provide easier analysis of the proposed representation, namely a faster perception of each point location, and a simple way of "grouping" the points belonging to the same comparison, when multiple comparison data is shown, the points are connected using line segments, forming a polygon (hereafter know as the difference polygon). This should not be interpreted as implying anatomical connection or proximity between articulators, but as a way to ease visualization. The difference polygon also allows reducing the size of the value dots, which leaves more space in the representation for adding other features, in the future.

\subsection{Directional Information}

One important aspect regarding the differences found when comparing vocal tract profiles is to characterise how that difference occurred from one configuration to the other, e.g., by a rise in the tongue dorsum or lowering of the velum. To provide such information, a vector is used, with origin in the parameter value and the direction of displacement. For the sake of simplicity, displacement direction is only represented for values corresponding to mild or strong differences (i.e., $<0.75$, according to the proposed interpretation thresholds). If the difference for a particular feature is deemed irrelevant (green circular corona), then representing a direction would have no meaning. Furthermore, it helps to highlight features depicting notable differences.

\subsection{Variability}

Since differences are often computed for multiple realizations of two sounds, and the average difference represented, it is also important to depict the variability associated with each value. To this end, several alternatives 
were tested such as bars and/or lines associated to each represented value, depicting data as in a boxplot, or a representation of dispersion outside the unitary circle. These tended to clutter the representation and became problematic if more that one difference polygon was represented.

Therefore, to provide variability data, the standard deviation for each parameter is represented over its direction and all parameter points used to draw an orange polygon (hereafter known as the deviation polygon) at the centre of the representation (figure $5 \mathrm{~g}$ ).

\section{Support for Analysis of Dynamic Aspects}

The previous sections proposed a method to compare between vocal tract contours and an abstract representation of the gathered data to support analysis, but leaving behind the assessment of dynamic aspects of speech production. Considering the proposed quantitative comparison methods, they can be extended to assess these dynamic aspects (Silva et al., 2013b; Silva and Teixeira, 2014b).

We propose a method for dynamic analysis of articulatory data jointly exploring multiple realisations of each sound and covering multiple articulators. Instead of analysing each utterance and then inferring notable features, all realisations for a particular utterance can be considered simultaneously, possibly from more than one speaker, aiming towards a characterisation of average dynamic aspects and their corresponding variation.

\subsection{Aligning Multiple Utterance Realisations}

Multiple realisations of the same utterance (or different utterances), identified by previously performed annotation (e.g., using Praat, Boersma and Weenink, 2014), commonly present different durations. Before performing any kind of analysis, taking advantage of multiple realisations to characterise the mean dynamic behaviour and dynamic intra- and inter-sound variability, they have to be properly matched.

The method used to perform the match can account for aspects such as the duration and complexity of the utterances and adapt accordingly. For example, for small utterance durations (or reduced number of phones), time axis normalization can be adopted, with each realisation's duration assumed to vary between 0 and 1 . Other approaches, when the utterances are longer or more complex, might include Dynamic Time Warping (DTW) (Berndt and Clifford, 1994). 
Based on the utterance realisations matching, the vocal tract contours extracted along the utterance are interpolated in order to allow comparison of corresponding contours between the realisations. Figure 6 shows an example of matching two realisations of [ [ ] using time axis normalization. One of the

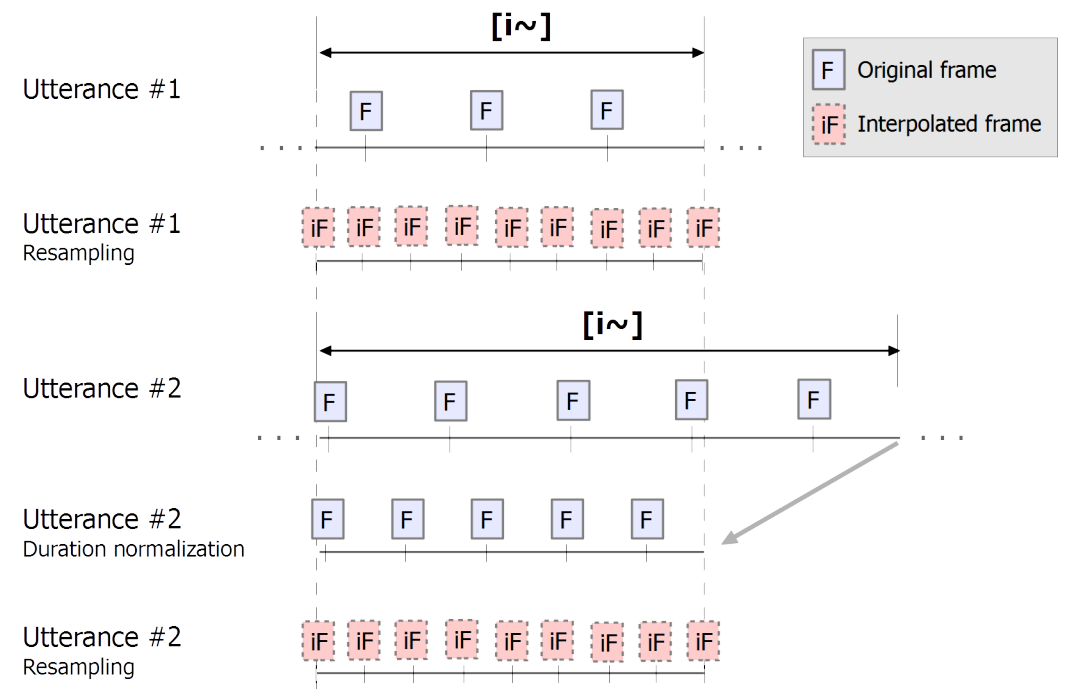

Figure 6: Aligning multiple realisations (or utterances) by normalizing duration. The duration of one realisation is used as reference and the corresponding image frames interpolated to obtain a fixed set of evenly distributed frames. The second realisation is compressed/stretched to the duration of the first and a similar interpolation procedure is applied enabling common time points for comparison.

realisations is considered as reference and the existing vocal tract contours (associated to the corresponding image frames) are interpolated to obtain 10 vocal tract configurations equally spaced along the realisation. The second realisation is assumed to last the same amount of time as the first and the existing vocal tract contours interpolated to obtain 10 configurations.

\subsection{Dynamic Analysis of the Vocal Tract}

After matching the different realisations for each utterance and obtaining the corresponding contours, the vocal tract comparison method presented earlier can be used to compare each contour pair along the production. Figure 7 depicts an illustrative example for the comparison between two sounds and considering 10 comparisons along the utterances' duration. Since several realisations are considered for each utterance there are multiple contours to 
consider at each time instant. For each of these comparisons the vocal tract contours of the two utterances are cross-compared (as detailed in section 2.4) and the standard deviation computed.

\subsection{Dynamic Data Representation}

Depicting dynamic data by representing the difference polygon for each vocal tract profile pair along the utterances (top rows in figure 7) is space consuming and can be difficult to grasp the full extent of the represented data. Therefore, we propose that the dynamic data is presented differently.

The representation of the data gathered for the dynamic analysis has four different parts as depicted, at the bottom, in figure 7 . On the left, a difference diagram for the comparison among the first frames and, on the right, a difference diagram for the comparison among the last frames of the considered utterances. The dynamic aspects are depicted in the middle. A line plot, with a line for each of the considered difference parameters, is provided. The line plot shows three horizontal colour bands as the difference diagrams, to hint on possible interpretations of the obtained values: as before, while green is proposed to stand for negligible differences, yellow and red stand for mild and strong differences. Since utterance matching has been performed, the time axis shows percentages instead of any particular time range. Below the central line plot, another line plot shows the standard deviation for each of the difference parameters.

If needed, more compact representations can be easily obtained, based on this first proposal, by not showing the difference diagrams for the first and last frame, only showing the standard deviation plot if it goes above a certain threshold, or just plotting the lines for features with notable variation.

\section{Application Examples}

In this section we show some examples of what can be accomplished using the framework, in its current state of development, by addressing different tasks relevant for articulatory analysis performed over an existing RT-MRI corpus for European Portuguese (EP). The main purpose is not to perform a detailed characterisation of the articulatory characteristics of $\mathrm{EP}$, or to fully explore the existing corpus, but to highlight those innovative aspects provided by the proposed quantitative analysis framework. 


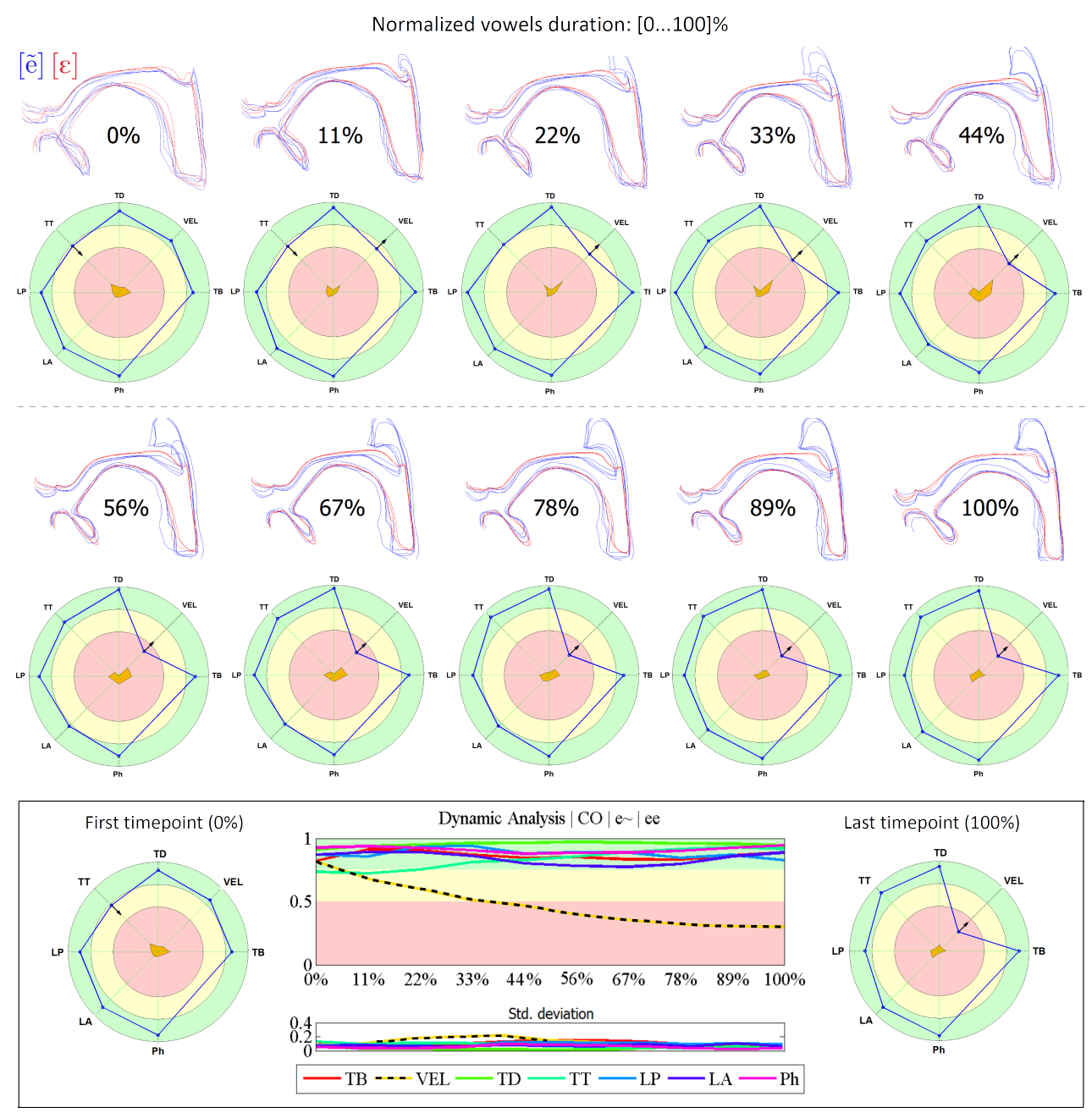

Figure 7: Extension of the comparison framework to cover vocal tract differences over time. Multiple realisations of $[\tilde{e}]$ and $[\varepsilon]$ are aligned by normalizing duration. At 10 points in time the vocal tract configurations are cross-compared between vowels resulting in comparison data along the vowels' duration. At the bottom, the comparison values obtained for each feature (e.g., velum (VEL), tongue tip (TT)) are presented, over time, using a line plot. 


\subsection{Application Scenario}

As subject we selected EP nasal vowels due to the availability of the data, its reported dynamic nature (Teixeira et al., 1999) and our group continued interest. The articulation of EP nasal vowels has been studied by the authors and colleagues mainly focusing on velar dynamics as provided by electromagnetic midsagittal articulography (EMMA) (Oliveira et al., 2009). To extend these studies, with a characterisation of the oral configuration of EP nasal vowels, static (Martins et al., 2008) and, more recently, real-time magnetic resonance imaging (RT-MRI) data of the vocal tract was acquired (Oliveira et al., 2012; Martins et al., 2012).This imaging modality provides adequate data regarding the position and coordination of the different articulators over time (Hagedorn et al., 2011) and might provide a good choice to tackle the hyperarticulation effect observed in sustained productions (Engwall, 2003). Additionally, it might also help reduce the gravity effect on articulators for acquisitions in supine position (Tiede and Vatikiotis-Bateson, 2000).

\subsubsection{Data Acquisition and Feature Extraction}

Image sequences were acquired containing: a) the five European Portuguese (EP) nasal vowels uttered in three word positions: initial, internal

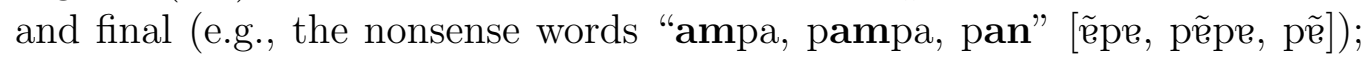
and b) the eight EP oral vowels (e.g., "papa" or "pupa").

Acquisition was performed at the midsagittal plane of the vocal tract. The images were acquired on an unmodified 3.0 T MR scanner (Magneton Tim Trio, Siemens, Erlanger, Germany) equipped with high performance gradients $(\operatorname{Gmax}=45 \mathrm{mT} / \mathrm{m}$, rise time $=0.2 \mathrm{~s}$, slew Rate $=200 \mathrm{~T} / \mathrm{m} / \mathrm{s}$, and FOV $=50 \mathrm{~cm}$ ). Custom 12-channel head and 4-channel neck phasedarray coils were used for data acquisition. Parallel imaging (GRAPPA 2) and magnetic field gradients operating at FAST mode were used to speed up the acquisition. After localisation images, a T1 W 2D-midsagittal MRI slice of the vocal tract was obtained, using an Ultra-Fast RF-spoiled Gradient Echo (GE) pulse sequence (Single-Shot TurboFLASH), with a slice thickness of $8 \mathrm{~mm}$ and the following parameters: TR/TE/FA $=72 \mathrm{~ms} / 1.02$ $\mathrm{ms} / 5^{\circ}$, Bandwidth $=1395 \mathrm{~Hz} /$ pixel, $\mathrm{FOV}\left(\mathrm{mm}^{2}\right)=210 \times 210$, reconstruction matrix of $(128 \times 128)$ elements with $50 \%$ phase resolution, in-plane resolution $\left(\mathrm{mm}^{2}\right)=3.3 \times 1.6$, yielding a frame rate of 14 images/second.

Typically, each recorded sequence contained 75 images (taking around 5 seconds to acquire) although some longer sequences (300 images) were also 
acquired, mostly with the speakers producing sequences of isolated vowels

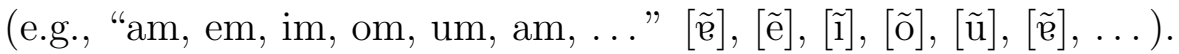

Audio was recorded simultaneously with the RT images inside the MR scanner, at a sampling rate of $16000 \mathrm{~Hz}$, using a fiberoptic microphone, and manually annotated using the software tool Praat (Boersma and Weenink, 2014), in order to identify the time intervals corresponding to different sounds. The time intervals allow the determination (because both data are aligned) of the corresponding image frames.

Data was acquired for three female speakers (CM, CO and SV), aged between 21 and 33, phonetically trained, with no history of hearing or speech disorders.

For additional details regarding the acquisition and corpus the reader is forwarded to Teixeira et al. (2012).

At an early stage of our research, vocal tract segmentation for this dataset was performed using a region-growing based method (Silva et al., 2013a) with the different segments of interest identified with the help of manually positioned landmarks, for each speaker (Silva et al., 2013b). Currently, the segmentation is handled by a model-based method (Silva and Teixeira, 2015) which results in a contour with the different segments of interest already tagged. Figure 8 shows some examples of image frames taken from the RTMRI dataset.

The examples that follow were computed gathering the data for each vowel irrespective of its context and considering the isolated vowels, which were not considered previously (Silva and Teixeira, 2014c,b).

\subsection{Static Analysis}

For the static analysis, since we are working with a real-time dataset, a representative contour of the configuration assumed by the vocal tract for each sound utterance is chosen. For example, based on previous experience with the considered dataset (Oliveira et al., 2012) we chose the middle frame for oral vowels and the final frame for nasal vowels. The different criterion was adopted since nasal vowels generally presented the lowest velum position at the end of the annotated segment. Nevertheless, any other criterion for choosing the vocal tract contours is possible since it does not influence how the differences are computed, but only how the resulting data is interpreted. 

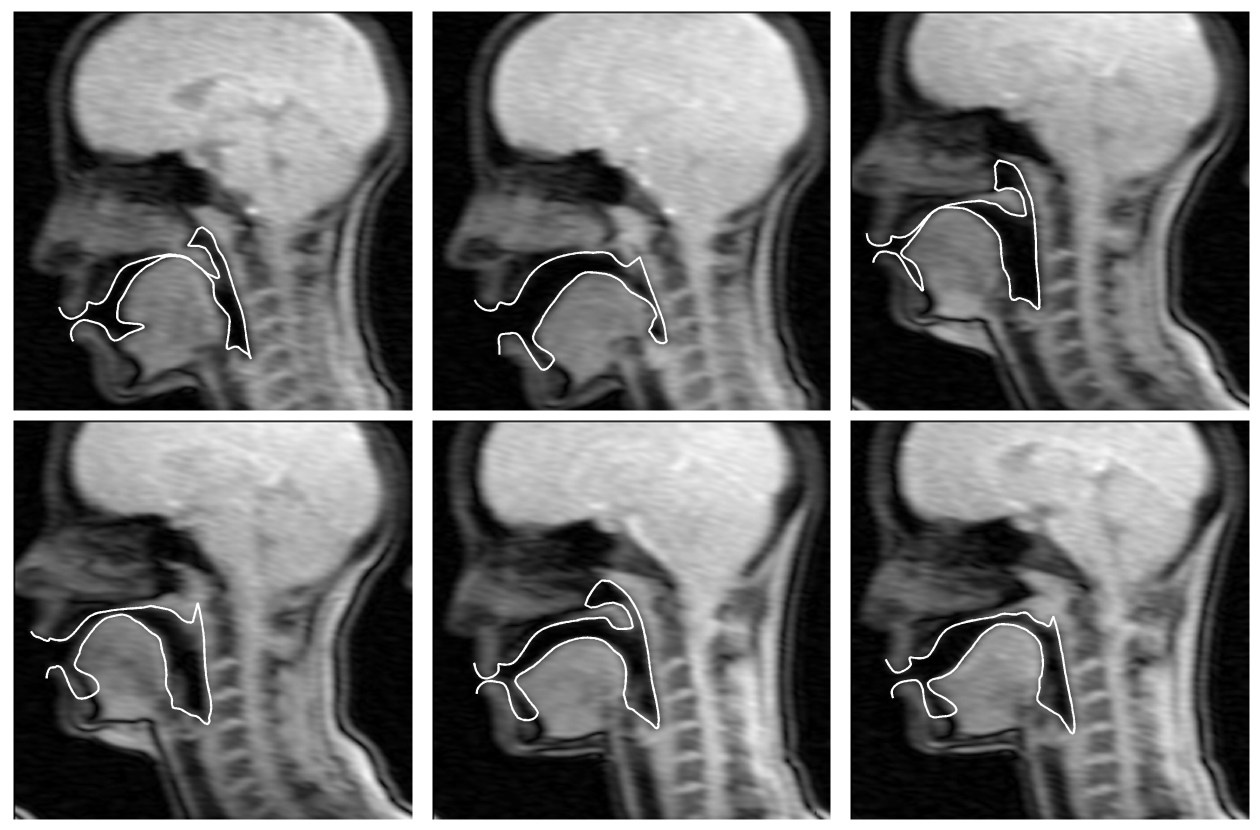

Figure 8: Sample images extracted from RT-MRI database acquired to study European Portuguese oral and nasal vowels and depicting the vocal tract contours.

\subsubsection{Intra-Speaker Analysis}

As already noted, multiple realisations of each vowel, produced by the same speaker, can be cross compared. This allows the assessment of the variability associated with the production of each vowel, for each speaker. Figure 9 presents some representative examples of the differences obtained for several vowels and speakers. In general, for the studied vowels, no major intra-vowel differences were found.

Quantitative analysis can also be performed to compare among vocal tract configurations for different sounds and for the same speaker. In EP, such comparisons are useful, for example, to study oral vs nasal vowels. Figure 10 depicts comparisons among different vowel pairs. The corresponding contour superpositions are also presented to allow observing how the computed data and abstract representation relate with the traditional visual assessment. To note, the clearly depicted differences: 1) at the velum between [i] and [i] and $[\tilde{u}]$ and $[u] ; 2$ ) in lip aperture, for all comparisons; and 3) at the tongue tip and tongue back, between $[\varepsilon]$ and $[\mathrm{e}]$.

Regarding the directional information, note that it should be read as an 

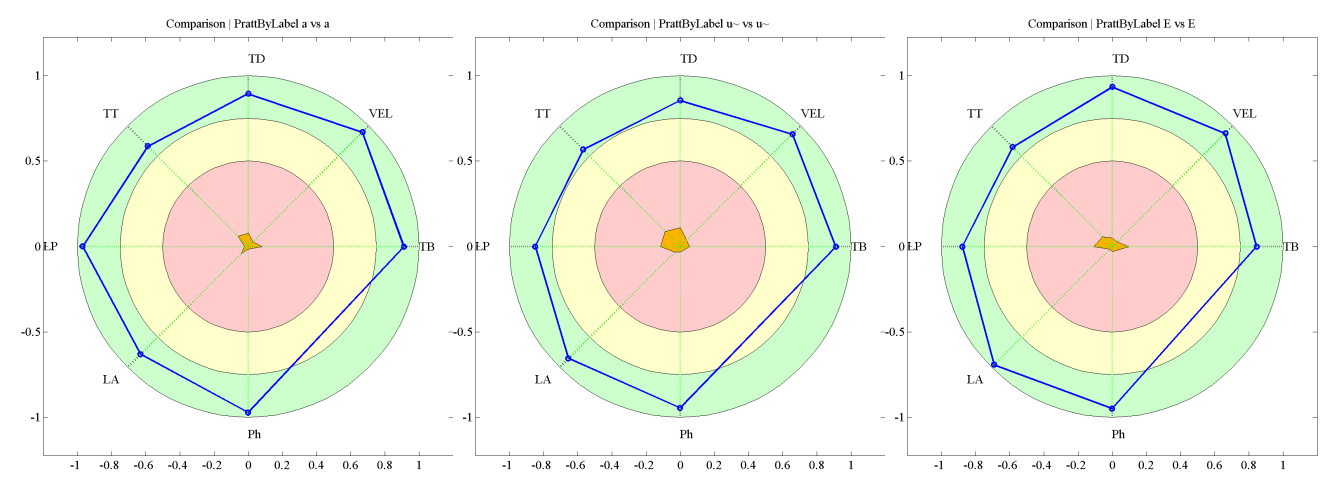

Figure 9: Vowel production variability per speaker obtained by cross-comparing all occurrences available. From left to right: [a] (speaker CM), [ũ] (speaker CO) and $[\varepsilon]$ (speaker $\mathrm{SV})$.

answer to the question "In which direction did the change occur from the first to the second vocal tract configuration?".

\subsubsection{Inter-Speaker Comparison}

One important feature provided by the proposed framework is the comparison among speakers. Since the provided comparison values are normalized, it is possible to visualise the differences for various speakers on a common representation. The difference polygons for each speaker and for each vowel pair were superimposed to assess inter-speaker differences (see figure 11). In general, the difference polygons for all speakers, for the same vowel pair, were similar. Notable inter-speaker differences were sometimes observed for lip aperture: notice, for example, for the comparison between [a] and [i], that speaker SV presents a mild difference for lip aperture.

\subsubsection{Overall characterisation of Articulatory Differences}

The data gathered for all speakers can now be used to assess the overall articulatory differences between vowels. Observing figure 12, a notable aspect, evidenced by the standard deviation, is that, overall, the strongest variability among the different productions is mostly observed at the tongue tip and lip aperture (protruding vertices of the orange polygon at the centre).

\subsection{Dynamic Analysis}

Considering the available data, three different kinds of dynamic analysis can be performed, covering the different articulators: 

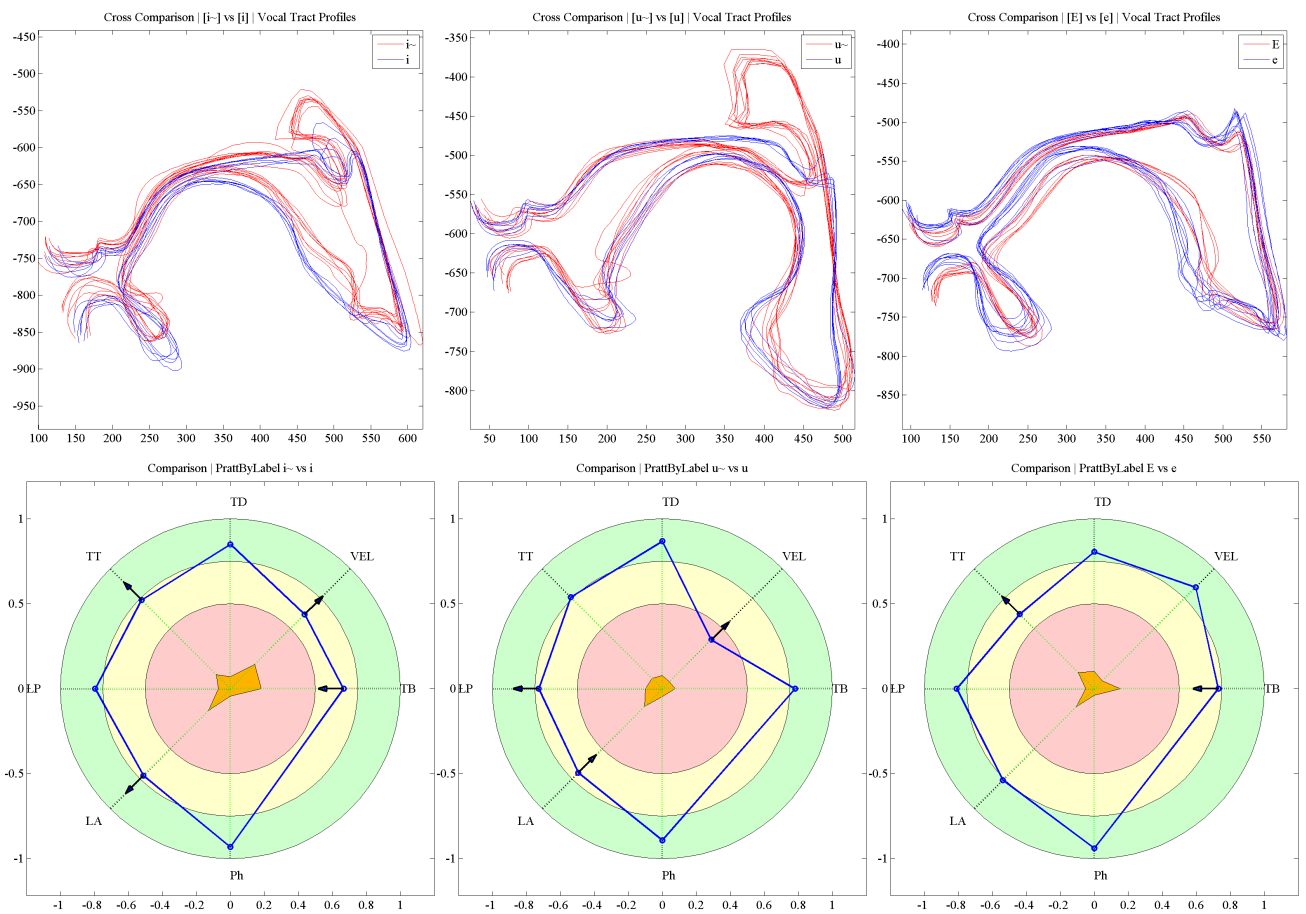

Figure 10: Comparison between the vocal tract configurations for two vowels for each speaker. The top row shows the considered vocal tract contours and the bottom row shows the correspondig difference diagram. From left to right: [ĩ] vs [i] (speaker CM), [ũ] vs $[\mathrm{u}]$ (speaker $\mathrm{CO}$ ) and $[\varepsilon]$ vs $[\mathrm{e}]$ (speaker $\mathrm{SV}$ ).

- Intra-sound dynamics, i.e., how does the vocal tract vary from the beginning to the end of the production and how consistent it is across productions. For example, cross compare all utterances of [i].

- Inter-sound comparison, i.e., compare the production of two different sounds along time to check differences/similarities. For example, vowel [o] and its nasal congener [õ].

- Comparison, over time, with a reference, i.e., analyse production of a sound using a particular (static) vocal tract configuration, as reference, to check how similar (and when) is the vocal tract configuration along

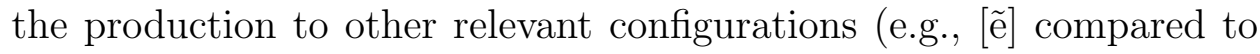
representative (static) configuration of $[\varepsilon]$ ). 

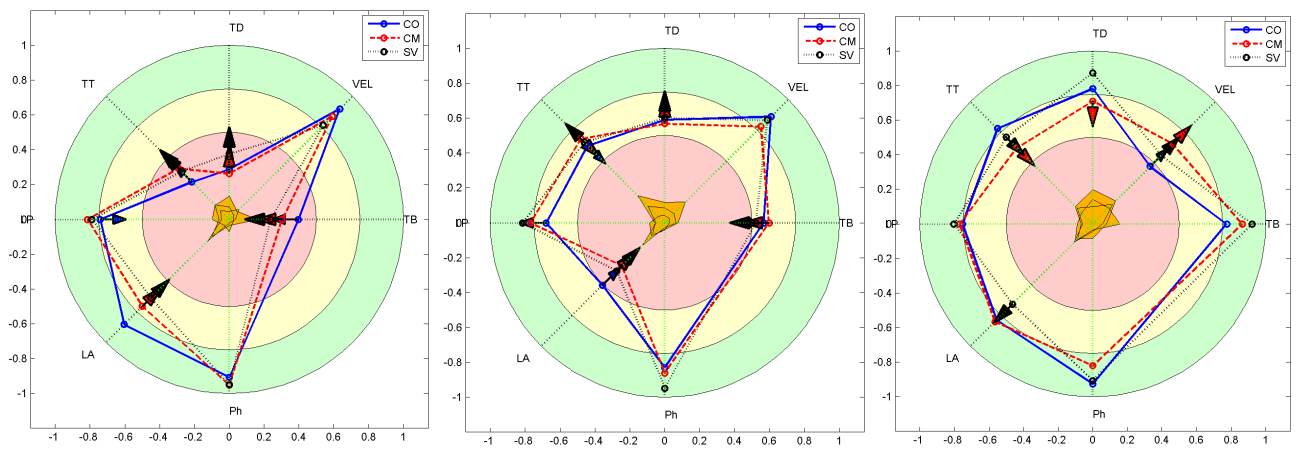

Figure 11: Inter-speaker comparison of differences between vowels. From left to right: [a] vs [i], [a] vs [u] and [õ] vs [ว].
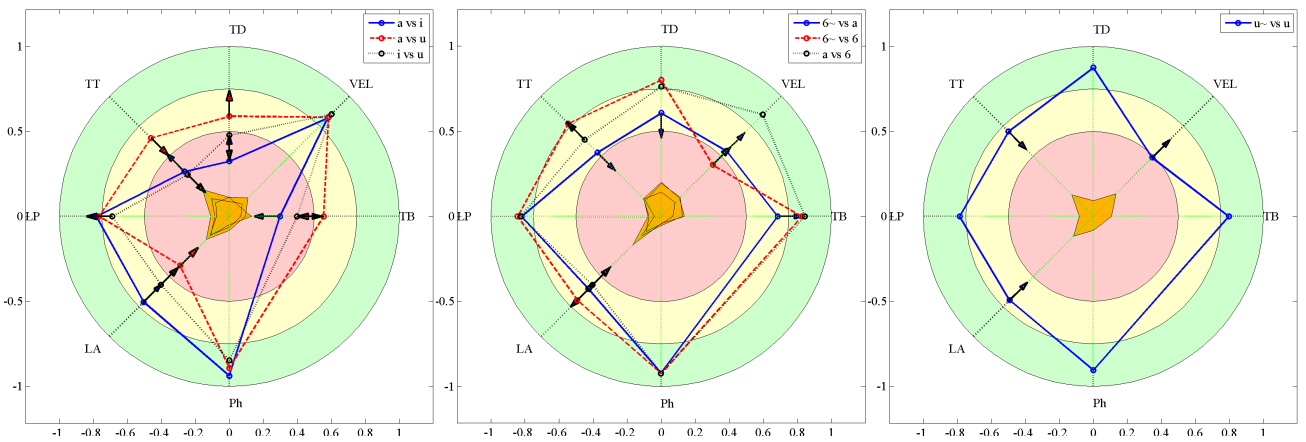

Figure 12: Differences among vowel pairs computed using data from all speakers. From left to right: $[\mathrm{a}][\mathrm{i}][\mathrm{u}],[\tilde{\mathrm{e}}][\mathrm{e}][\mathrm{a}]$ and $[\tilde{u}][\mathrm{u}]$.

\subsubsection{Intra-sound Dynamics}

All utterances of a particular vowel are matched and cross compared. The resulting comparison data for each considered frame is averaged and represented in the line plot to depict evolution over time. Figure 13 shows, as representative examples, variation data for vowels $[\tilde{\mathrm{e}}]$ and $[\tilde{\mathrm{u}}]$. For $[\tilde{\mathrm{e}}]$ only data from speaker CM was considered, but since all utterances can be matched and the vocal tract comparison method provides normalized data, the presented dynamic analysis for vowel [ũ] was performed taking into account the data for all three speakers in the RT-MRI dataset. Visualizations made possible by the proposed method show no major discrepancy of vocal tract configuration across utterances, for both vowels, i.e., all utterances exhibit a similar vocal tract variation pattern: a slight difference is observed for the lip aperture (LA), in vowel $[\tilde{\mathrm{e}}]$, at the beginning and end of the 


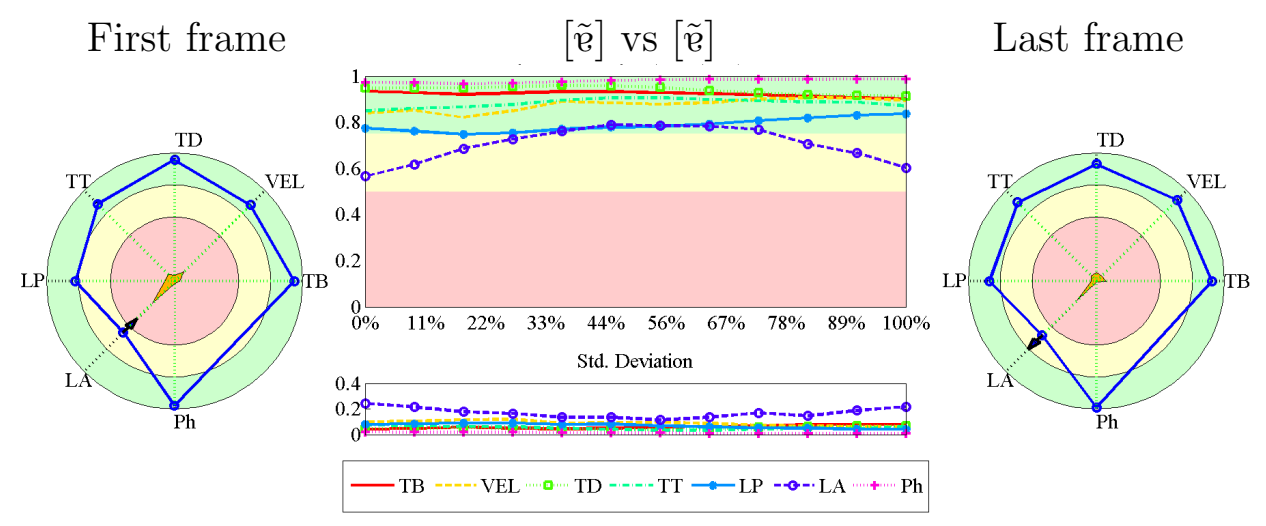

First frame

$[\tilde{u}]$ vs $[\tilde{u}]$

Last frame
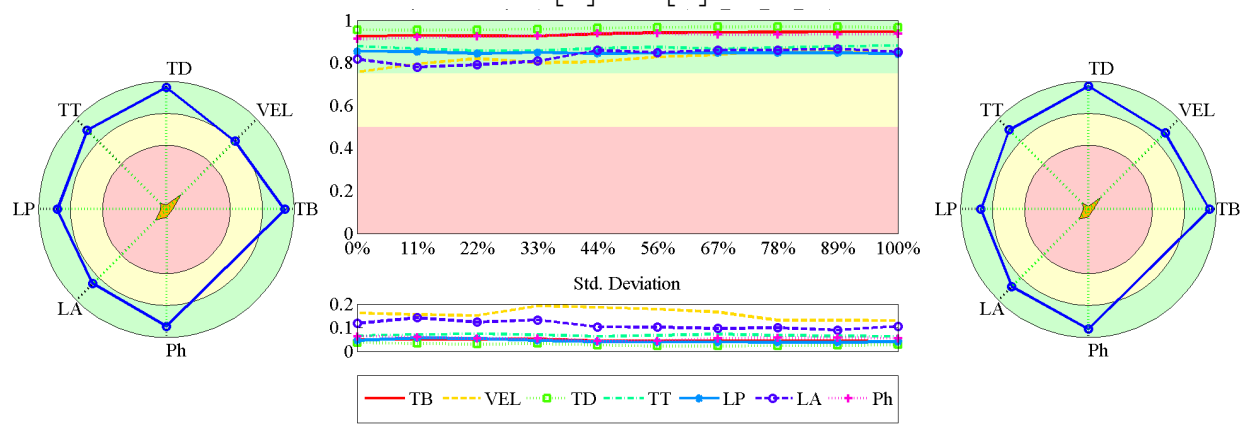

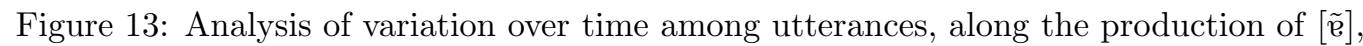
for speaker CM (top) and [ũ] for all speakers (bottom).

production, but the remaining parameters keep above 0.75 with low standard deviations.

\subsubsection{Inter-sound production differences}

Figure 14 shows two examples regarding the assessment of dynamic differences between sounds. In the first example (top row), the production of $[\tilde{e}]$ was compared with the production of $[\varepsilon]$ : both start very similar with only a slight difference at tongue tip and gradually differ at lip aperture and velum. This gradual variation at the velum is in agreement with previous studies (Gregio (2006); Lovatto et al. (2007); Martins et al. (2012); Oliveira et al. (2012)) describing different phases in the production of nasal vowels.

The second example (figure 14, bottom row) shows the comparison between vowels $[\tilde{u}]$ and $[\tilde{1}]$. As expected there are strong differences at the 


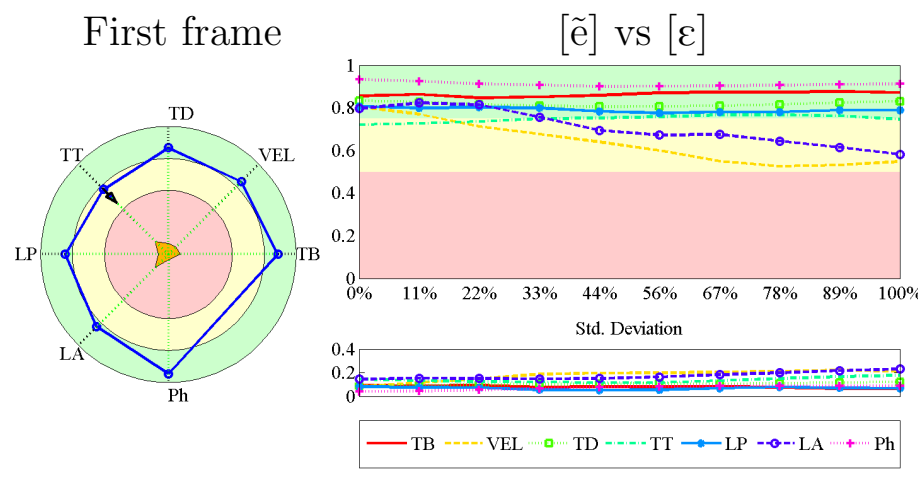

Last frame
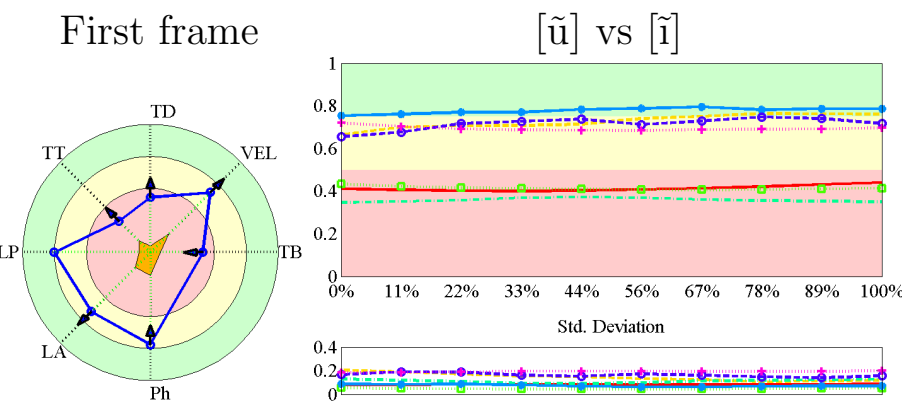

Last frame

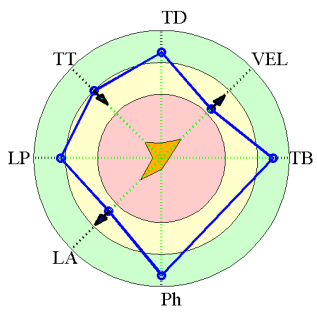

-

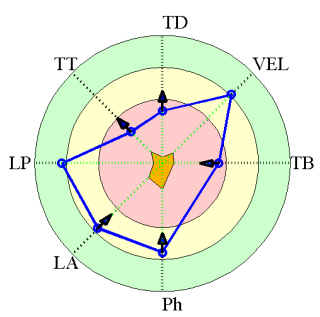

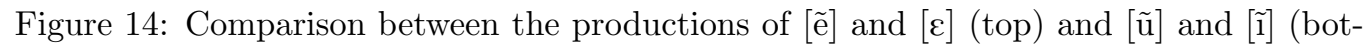
tom). Analysis performed considering data from all speakers.

tongue tip (TT), tongue back (TB) and tongue dorsum (TD). Nevertheless, note how no substantial difference exists at the velum (VEL) between the two sounds. Since both are nasal vowels, the velum is expected to gradually open along the production (Martins et al., 2012). The dynamic analysis shows that both sounds exhibit a similar velum aperture pattern.

\section{Discussion}

Taking into consideration the challenges identified in the introduction, we consider that the proposed framework provides important contributions: addresses subjectivity by proposing quantitative methods for comparing vocal tract profiles; allows comparing data among speakers due to the normalized nature of the computed differences; makes it possible to gather the data from different realisations of each sound in order to compute variability; and 
supports gathering data from all available speakers to depict overall characterisation of articulatory differences, e.g., for a particular language.

The application examples presented show how the proposed methods can be useful to tackle the analysis of large datasets and provide data enabling the characterisation of the articulatory differences between different sounds both considering static and dynamic analysis.

The only challenge that we do not explicitly address, from those pointed in the introduction, is multimodality. It is our understanding that the defined framework is general enough to allow its extension to encompass data from other modalities. Nevertheless, it is important to note that this extension poses interesting new challenges such as how to deal with the different number of features covered by each modality (e.g., the tongue in US and the whole vocal tract in MRI) or how to consider the contributions of each modality to the presented average data.

In a systematic, unsupervised approach to the analysis of articulatory data (e.g., considering our pipeline in figure 1), it is possible that, due to image noise or artefacts, the quality of the unsupervised vocal tract segmentation (Silva and Teixeira, 2015) might sometimes be compromised (Ramanarayanan et al., 2013). The inclusion of such segmentations in the analysis (albeit in small number) might eventually affect the results and the pipeline would profit from methods to filter the data or signal these problematic cases for further analysis. One possible approach, a relevant additional application made possible by the proposed framework, might be the detection of outliers based on intra-sound comparisons.

The features proposed for this first instantiation of the framework, and the methods used to compute them, are a first proposal and can be subject to changes and improvements. For example, comparison of regions using the Pratt index is performed by determining the corresponding point by a proximity criterion. This might be improved by searching for the corresponding points along the expected principal direction of movement, e.g., radial, for tongue dorsum. Regarding the velum, if the available vocal tract contours allow analysis of velar aperture, adding to the velum position (e.g., Silva and Teixeira, 2015), this data can be considered for the computation of $\mathbf{F}_{V E L}$. Nevertheless, despite the possible improvements and changes to the methods used to compute each feature, it is important to note that the proposed framework allows systematic and objective application of an uniform comparison criteria throughout the compared data.

Furthermore, the proposed comparison features are not to be understood 
as a closed set. For this first instantiation of the framework, we chose a set of features that, overall, cover the different articulators, but new features (e.g., Saltzman and Munhall, 1989; Smith, 2014) can be added to better serve the study of specific sounds. For example, considering features that deal with constriction degree and location would allow the study of consonantal constriction.

One of the aspects we consider relevant for the framework is the possibility to depict the comparison outcomes in such a way that it is easily interpreted by researchers. The abstract visual representations are a first proposal to help gather insight over the computed comparison values and, even though simple in nature, serve their main purpose well, as demonstrated in the application examples. Nevertheless, these representations present some limitations. When multiple difference polygons are represented and, hence, multiple polygons depicting the corresponding standard deviations, there is overlap among the polygons that might hinder the interpretation: matching between the difference polygons and the corresponding standard deviations is not possible and the larger polygon might cover the remaining polygons, if drawn last. Nonetheless, this already provides an idea of the worst case scenario. We have been gathering feedback from different researchers concerning the proposed representations, a first informal evaluation was presented in Silva and Teixeira (2014a) and we intend to further explore user inputs.

It is important to note that using the proposed framework results in a set of quantitative difference data that can be further explored for computational analysis. The proposal of the visual representations should not be understood as the final stage for the computed comparison data. Although not explored in this article, methods such as clustering can be tested to study the proximity between sounds, speakers or languages and the importance of particular features in differencing between sounds.

\section{Conclusions}

This article proposes a quantitative framework for vocal tract profile comparison. To the best of our knowledge, this is the first time a quantitative analysis framework is presented encompassing the assessment of per sound intra-speaker variability, inter-speaker differences and overall inter-sound differences using data from all speakers in a single representation. The presented application examples show the potential of the proposed framework to take advantage of the increasing amounts of articulatory data available and move 
towards quantitative inter-speaker and inter-language comparison, both for static and dynamic analysis scenarios.

The application of the proposed methods to other classes of sounds (e.g., laterals, Martins et al., 2010) and the study (and inclusion in the proposed visualisations) of landmark trajectories and their variability, considering all available speakers, should provide further insight into the usefulness of the proposed methods.

Adding to the possible improvements already mentioned in the discussion, there are a few other lines of work that deserve further attention. Grounded on the principles and methods encompassed by the proposed framework, promoting systematic analysis of the available vocal tract data, the presented work can evolve to provide support to the use of high-level models of articulator organization and control (e.g., TAsk Dynamics Application (TADA), Nam et al., 2015). Regarding the identification of relevant gestures, the analysis based on multiple realisations of each sound can already be helpful, and the instantiation of the framework considering other features (e.g., tongue body and tip constriction location and degree) can bring the outcomes closer to the variables required by the models. The aspect that needs to be further developed is the computation of temporal aspects that, despite being present in the dynamic analysis, is not explicitly addressed. In this regard, the longterm goal is the generation of gestural scores, from the data, that can serve as input, for example, to articulatory synthesizers (e.g., Teixeira et al., 2002; Birkholz et al., 2011)

The use of multi-planar (e.g., Proctor et al., 2012) or 3D imaging (e.g., Martins et al., 2011; Zhu et al., 2013) of the vocal tract assumes importance for studying sounds exhibiting important characteristics not observable in the sagittal plane (Zhu et al., 2013). While the work presented here does not explicitly address the application of the framework to these kind of MRI data, there is no impediment to it, as long as the new features comply with the requirements set in section 2 and analysis follows the systematic procedure inherent to the framework. Instead of one midsagittal vocal tract contour, several contours can be considered, in different planes of interest. For instance, regarding lateral sounds (e.g., /l/), a comparison feature might be used accounting for the asymmetric nature of the lateral channels forming on the tongue sides (Martins et al., 2010), based on their section area, in the coronal plane, and their length.

One route we are considering for further development of the proposed framework, addressing the multimodality challenge discussed earlier, is its 
use with data from different/multiple imaging modalities. We are currently considering the analysis of RT-MRI and ultrasound data simultaneously.

The presented methods (and envisaged steps regarding computational analysis) are computationally demanding and generate large amounts of data. Therefore, their deployment in a cloud environment would provide a more suitable scenario for further developments and an important first step towards their validation and use by third parties such as phoneticians. The use of the proposed framework and its developments by other research groups, over their data, would pave the way for comparisons between dialects and languages. We are currently starting this migration process in the scope of projects Cloud Thinking ${ }^{1}$ and IRIS $^{2}$.

\section{Acknowledgements}

The authors thank the anonymous reviewers for their helpful comments and suggestions. Research partially funded by FEDER through the Program COMPETE and by National Funds (FCT) in the context of HERON II (PTDC/EEAPLP/098298/2008), Project Marie Curie IAPP "IRIS" (FP7-PEOPLE-2013IAPP, ref. 610986) and project Cloud Thinking (QREN Mais Centro, ref. CENTRO-07-ST24-FEDER-002031).

\section{References}

Badin, P., Sawallis, T.R., Crépel, S., Lamalle, L., 2014. Comparison of articulatory strategies for bilingual speaker: Preliminary data and models, in: Proc. ISSP, Cologne, Germany. pp. 17-20.

Benítez, A., Ramanarayanan, V., Goldstein, L., Narayanan, S., 2014. Realtime MRI of articulatory setting in second language speech, in: Proc. Interspeech, Singapore. pp. 701-705.

Berndt, D., Clifford, J., 1994. Using dynamic time warping to find patterns in time series, in: Proc. AAAI Workshop on Knowledge Discovery in Databases, pp. 229-248.

\footnotetext{
${ }^{1}$ http://cloudthinking.web.ua.pt/ieeta/

${ }^{2}$ www.microsoft.com/pt-pt/mldc/iris/default.aspx
} 
Birkholz, P., Kroger, B., Neuschaefer-Rube, C., 2011. Model-based reproduction of articulatory trajectories for consonant-vowel sequences. IEEE Transactions on Audio, Speech, and Language Processing 19, 1422-1433. doi:10.1109/TASL . 2010.2091632.

Boersma, P., Weenink, D., 2014. Praat: doing phonetics by computer [computer program]. version 5.3.42. URL: http://www.praat.org/.

Bresch, E., Katsamanis, A., Goldstein, L., Narayanan, S., 2010. Statistical multi-stream modeling of real-time MRI articulatory speech data, in: Proc. Interspeech, Makuhari, Japan. pp. 1584-1587.

Bresch, E., Narayanan, S., 2009. Region segmentation in the frequency domain applied to upper airway Real-Time Magnetic Resonance images. IEEE Transactions on Medical Imaging 28, 323-338. doi:10.1109/TMI. 2008.928920 .

Cleland, J., Wrench, A.A., Scobbie, J.M., Semple, S., 2011. Comparing articulatory images: An MRI / ultrasound tongue image database, in: Proc. Int. Seminar on Speech Production (ISSP), Montreal, Canada. pp. 163-170.

Davidson, L., 2006. Comparing tongue shapes from ultrasound imaging using smoothing spline analysis of variance. Journal of the Acoustical Society of America 120, 407-415.

Delvaux, V., Metens, T., Soquet, A., 2002. Propriétés acoustiques e articulatoires des voyelles nasales du Français, in: Proc. Journées d'Étude sur la Parole, Nancy. pp. 348-352.

Engwall, O., 2003. A revisit to the application of MRI to the analysis of speech production - testing our assumptions, in: Proc. 6th Int. Seminar on Speech Production (ISSP), Sydney, Australia. pp. 43-48.

Gick, B., Wilson, I., Koch, K., Cook, C., 2004. Language-specific articulatory seetings: Evidence from inter-utterance rest position. Phonetica 61, 220233.

Gregio, F.N., 2006. Configuração do trato vocal supraglótico na produção das vogais do Português Brasileiro: dados de imagens de ressonância magnética 
[Supraglottic vocal tract shaping in the production of Brazilian Portuguese vowels: data from magnetic resonance imaging]. Master thesis. PUC/SP.

Hagedorn, C., Proctor, M.I., Goldstein, L., 2011. Automatic analysis of singleton and geminate consonant articulation using Real-Time Magnetic Resonance Imaging, in: Proc. Interspeech, Florence, Italy. pp. 409-412.

Höwing, F., Dooley, S., Wermser, D., 1999. Tracking of non-rigid articulatory organs in X-ray image sequences. Computerized Medical Imaging and Graphics 23, 59-67.

Kim, J., Lammert, A., Kumar Ghosh, P., Narayanan, S., 2014. Coregistration of speech production datasets from electromagnetic articulography and real-time magnetic resonance imaging. The Journal of the Acoustical Society of America 135, EL115-EL121. doi:10.1121/1. 4862880 .

Lammert, A., Proctor, M., Narayanan, S., 2010. Data-driven analysis of realtime vocal tract MRI using correlated image regions, in: Proc. Interspeech, Makuhari, Japan. pp. 1572-1575.

Laprie, Y., Aron, M., Berger, M.O., Wrobel-Dautcourt, B., 2014. Studying MRI acquisition protocols of sustained sounds with a multimodal acquisition system, in: Proc. $10^{\text {th }}$ Int. Seminar on Speech Production (ISSP), Cologne, Germany. pp. 245-248.

Lovatto, L., Amelot, A., Crevier-Buchman, L., Basset, P., Vaissière, J., 2007. A fiberscopic analysis of nasal vowels in Brazilian Portuguese, in: Proc. 16th International Congress of Phonetic Sciences (ICPhS), Saarbrücken, Germany. pp. 549-552.

Martins, P., Carbone, I., Pinto, A., Silva, A., Teixeira, A., 2008. European Portuguese MRI based speech production studies. Speech Communication 50, 925-952.

Martins, P., Oliveira, C., Silva, A., Teixeira, A., 2010. Articulatory characteristics of European Portuguese laterals: a 2D \& 3D MRI study, in: Proc. FALA, Vigo, Spain. pp. 33-36.

Martins, P., Oliveira, C., Silva, S., Silva, A., Teixeira, A., 2011. Tongue segmentations from mri images using itk-snap: Preliminary evaluation, 
in: Proc. IADIS Int.l Conferences on Computer Graphics, Visualization, Computer Vision and Image Processing.

Martins, P., Oliveira, C., Silva, S., Teixeira, A., 2012. Velar movement in European Portuguese nasal vowels, in: Proc. IberSpeech 2012 - VII Jornadas en Tecnología del Habla and III Iberian SLTech Workshop, Madrid, Spain. pp. 231-240.

Miller, N.A., Gregory, J.S., Aspden, R.M., Stollery, P.J., Gilbert, F.J., 2014. Using active shape modeling based on MRI to study morphologic and pitch-related functional changes affecting vocal structures and the airway. Journal of Voice [in press]. doi:10.1016/j.jvoice.2013.12.002.

Nam, H., Browman, C., Goldstein, L., Proctor, M., Rubin, P., Saltzman, E., 2015. TADA: TAsk Dynamic Application. URL: http://www.haskins . yale.edu/tada_download/index.php.

Niebergall, A., Zhang, S., Kunay, E., Keydana, G., Job, M., Uecker, M., Frahm, J., 2013. Real-time MRI of speaking at a resolution of $33 \mathrm{~ms}$ : Undersampled radial FLASH with nonlinear inverse reconstruction. Magnetic Resonance in Medicine 69, 477-485.

Oliveira, C., Martins, P., Silva, S., Teixeira, A., 2012. An MRI study of the oral articulation of European Portuguese nasal vowels, in: Proc. Interspeech, Portland, Oregon, USA. pp. 2690-2693.

Oliveira, C., Martins, P., Teixeira, A., 2009. Speech rate effects on European Portuguese nasal vowels, in: Proc. Interspeech, Brighton, UK. pp. 480-483.

Pratt, W.K., 2007. Digital Image Processing. Wiley-Interscience.

Proctor, M., Bone, D., Katsamanis, A., Narayanan, S., 2010. Rapid semiautomatic segmentation of real-time magnetic resonance images for parametric vocal tract analysis, in: Proc. Interspeech, Makuhari, Japan. pp. 1576-1579.

Proctor, M., Lu, L.H., Zhu, Y., Goldstein, L., Narayanan, S., 2012. Articulation of Mandarin sibilants: a multi-plane realtime MRI study, in: Proc. Speech Sci. Tech, Sydney, Australia. pp. 113-116. 
Proctor, M.I., Lammert, A.C., Katsamanis, A., Goldstein, L.M., Hagedorn, C., Narayanan, S.S., 2011. Direct estimation of articulatory kinematics from real-time magnetic resonance image sequences, in: Proc. Interspeech, Florence, Italy. pp. 281-284.

Ramanarayanan, V., Byrd, D., Goldstein, L., Narayanan, S.S., 2010. Investigating articulatory setting - pauses, ready position, and rest - using real-time MRI, in: Proc. Interspeech, Makuhari, Japan. pp. 1994-1997.

Ramanarayanan, V., Goldstein, L., Byrd, D., Narayanan, S., 2013. An investigation of articulatory setting using real-time magnetic resonance. Journal of the Acoustical Society of America 134, 510-519.

Saltzman, E., Munhall, K., 1989. A dynamical approach to gestural paterning in speech production. Ecological Psychology 1, 333-382.

Scott, A.D., Wylezinska, M., Birch, M.J., Miquel, M.E., 2014. Speech MRI: Morphology and function. Physica Medica [in press]. doi:10.1016/j. ejmp.2014.05.001.

Shadle, C., Proctor, M.I., Iskarous, K., 2008. An MRI study of the effect of vowel context on English fricatives, in: Proc. Acoustics '08 Paris: Joint meeting of the ASA, EAA \& Société Française d'Acoustique.

Shosted, R., Sutton, B.P., Benmamoun, A., 2012. Using magnetic resonance to image the pharynx during Arabic speech: Static and dynamic aspects, in: Proc. Interspeech, Portland, Oregon, USA. pp. 2182-2185.

Silva, S., Teixeira, A., 2014a. A framework for analysis of the upper airway from real-time MRI sequences, in: Proc. SPIE Visualization and Data Analisis, SF, CA, USA. p. 901703. doi:10.1117/12.2042081.

Silva, S., Teixeira, A., 2014b. RT-MRI based dynamic analysis of vocal tract configurations: Preliminary work regarding intra- and inter-sound variability, in: Proc. Int. Seminar on Speech Production (ISSP), Cologne, Germany. pp. 399-402.

Silva, S., Teixeira, A., 2014c. Systematic and quantitative analysis of vocal tract data: Intra- and inter-speaker analysis, in: Proc. Int. Seminar on Speech Production (ISSP), Cologne, Germany. pp. 403-406. 
Silva, S., Teixeira, A., 2015. Unsupervised segmentation of the vocal tract from real-time mri sequences. Computer Speech and Language 33, 25-46. doi:10.1016/j.csl.2014.12.003.

Silva, S., Teixeira, A., Oliveira, C., Martins, 2013a. Segmentation and analysis of vocal tract from midsagittal real-time MRI, in: Proc. ICIAR 2013, LNCS vol. 7950, Póvoa de Varzim, Portugal. pp. 459-466.

Silva, S., Teixeira, A., Oliveira, C., Martins, P., 2013b. Towards a systematic and quantitative analysis of vocal tract data, in: Proc. Interspeech, Lyon, France. pp. 1307-1311.

Smith, C., 2014. Complex tongue shaping in lateral liquid production without constriction-based goals, in: Proc. ISSP, Cologne, Germany. pp. 413-416.

Teixeira, A., Martins, P., Oliveira, C., Ferreira, C., Silva, A., Shosted, R., 2012. Real-time MRI for Portuguese: database, methods and applications, in: Proc PROPOR 2012, LNCS vol. 7243, Coimbra, Portugal. pp. 306-317.

Teixeira, A., Martins, P., Silva, A., Oliveira, C., 2011. An MRI study of consonantal coarticulation resistance in Portuguese, in: Proc. 9th Int. Seminar on Speech Production (ISSP), Montreal, Canada. pp. 243-250.

Teixeira, A., Silva, L., Martinez, R., Vaz, F., 2002. Sapwindows - towards a versatile modular articulatory synthesizer, in: Proc. IEEE Workshop on Speech Synthesis, pp. 31-34. doi:10.1109/WSS.2002.1224366.

Teixeira, A., Vaz, F., Príncipe, J.C., 1999. Influence of dynamics in the perceived naturalness of portuguese nasal vowels, in: Proc. Int. Cong. Phonetic Sciences (ICPhS), San Francisco, CA, USA. pp. 2557-2560.

Tiede, M.K., Vatikiotis-Bateson, E., 2000. Contrasts in speech articulation observed in sitting and supine conditions, in: Proc. 5th Seminar on Speech Production (ISSP), Chiemgau, Germany. pp. 25-28.

Wang, Y., 2011. Smooting Splines: Methods and Applications. CRC Press.

Zharkova, N., Hewlett, N., 2009. Measuring lingual coarticulation from midsagittal tongue contours: Description and example calculations using english /t/ and /a/. Journal of Phonetics 37, 248 - 256. doi:10.1016/j. wocn. 2008.10.005. 
Zharkova, N., Hewlett, N., Hardcastle, W.J., 2011. Coarticulation as an indicator of speech motor control development in children: An ultrasound study. Motor Control 15, 118-140.

Zharkova, N., Hewlett, N., Hardcastle, W.J., Lickley, R.J., 2014. Spatial and temporal lingual coarticulation and motor control in preadolescents. Journal of Speech, Language \& Hearing Research 57, 374-388.

Zhu, Y., Toutios, A., Narayanan, S., Nayak, K., 2013. Faster 3D vocal tract real-time MRI using constrained reconstruction, in: Proc. Interspeech, Lyon, France. pp. 1292-1296. 